\title{
Global Dynamics and Bifurcations Analysis of a Two-Dimensional Discrete-Time Lotka-Volterra Model
}

\author{
A. Q. Khan $(1)$ and M. N. Qureshi \\ Department of Mathematics, University of Azad Jammu and Kashmir, Muzaffarabad 13100, Pakistan \\ Correspondence should be addressed to A. Q. Khan; abdulqadeerkhan1@gmail.com
}

Received 23 August 2017; Revised 11 December 2017; Accepted 19 December 2017; Published 21 January 2018

Academic Editor: Abraham J. A. Tawil

Copyright (C) 2018 A. Q. Khan and M. N. Qureshi. This is an open access article distributed under the Creative Commons Attribution License, which permits unrestricted use, distribution, and reproduction in any medium, provided the original work is properly cited.

In this paper, global dynamics and bifurcations of a two-dimensional discrete-time Lotka-Volterra model have been studied in the closed first quadrant $\mathbb{R}^{2}$. It is proved that the discrete model has three boundary equilibria and one unique positive equilibrium under certain parametric conditions. We have investigated the local stability of boundary equilibria $O(0,0), A\left(\left(\alpha_{1}-1\right) / \alpha_{3}, 0\right)$, $B\left(0,\left(\alpha_{4}-1\right) / \alpha_{6}\right)$ and the unique positive equilibrium $C\left(\left(\left(\alpha_{1}-1\right) \alpha_{6}-\alpha_{2}\left(\alpha_{4}-1\right)\right) /\left(\alpha_{3} \alpha_{6}-\alpha_{2} \alpha_{5}\right),\left(\alpha_{3}\left(\alpha_{4}-1\right)+\alpha_{5}\left(1-\alpha_{1}\right)\right) /\left(\alpha_{3} \alpha_{6}-\alpha_{2} \alpha_{5}\right)\right)$, by the method of linearization. It is proved that the discrete model undergoes a period-doubling bifurcation in a small neighborhood of boundary equilibria $A\left(\left(\alpha_{1}-1\right) / \alpha_{3}, 0\right), B\left(0,\left(\alpha_{4}-1\right) / \alpha_{6}\right)$ and a Neimark-Sacker bifurcation in a small neighborhood of the unique positive equilibrium $C\left(\left(\left(\alpha_{1}-1\right) \alpha_{6}-\alpha_{2}\left(\alpha_{4}-1\right)\right) /\left(\alpha_{3} \alpha_{6}-\alpha_{2} \alpha_{5}\right),\left(\alpha_{3}\left(\alpha_{4}-1\right)+\alpha_{5}\left(1-\alpha_{1}\right)\right) /\left(\alpha_{3} \alpha_{6}-\alpha_{2} \alpha_{5}\right)\right)$. Further it is shown that every positive solution of the discrete model is bounded and the set $\left[0, \alpha_{1} / \alpha_{3}\right] \times\left[0, \alpha_{4} / \alpha_{6}\right]$ is an invariant rectangle. It is proved that if $\alpha_{1}<1$ and $\alpha_{4}<1$, then equilibrium $O(0,0)$ of the discrete model is a global attractor. Finally it is proved that the unique positive equilibrium $C\left(\left(\left(\alpha_{1}-1\right) \alpha_{6}-\alpha_{2}\left(\alpha_{4}-1\right)\right) /\left(\alpha_{3} \alpha_{6}-\alpha_{2} \alpha_{5}\right),\left(\alpha_{3}\left(\alpha_{4}-1\right)+\alpha_{5}\left(1-\alpha_{1}\right)\right) /\left(\alpha_{3} \alpha_{6}-\alpha_{2} \alpha_{5}\right)\right)$ is a global attractor. Some numerical simulations are presented to illustrate theoretical results.

\section{Introduction}

In this paper, we study the global dynamics and bifurcations analysis of a two-dimensional discrete-time Lotka-Volterra model in the closed first quadrant $\mathbb{R}^{2}$, which was proposed by Waltman [1]. In this model, if two populations are growing logistically without affecting each other, then their growth can be represented by the following system of two logistic equations:

$$
\begin{aligned}
& \frac{d x}{d t}=r_{1} x\left(1-\frac{x}{k_{1}}\right) \\
& \frac{d y}{d t}=r_{2} y\left(1-\frac{y}{k_{2}}\right),
\end{aligned}
$$

where $r_{1}, r_{2}, k_{1}, k_{2}$ and the initial conditions $x_{0}, y_{0}$ are positive real numbers. Now, assume that the carrying capacity is a shared resource-each population competes for the resource and thereby interferes with the other. Then the presence of each reduces the intrinsic rate of growth of the other. We refer the reader to [1-5] for detailed discussion on the above assumption. This phenomena can be represented as follows:

$$
\begin{aligned}
& \frac{d x}{d t}=r_{1} x\left(1-\frac{x}{k_{1}}-\zeta_{1} y\right) \\
& \frac{d y}{d t}=r_{2} y\left(1-\frac{y}{k_{2}}-\zeta_{2} x\right),
\end{aligned}
$$

where $\zeta_{1}, \zeta_{2}$ are positive constants. It is convenient to change the nondimensional variables by measuring $x$ in units of $k_{1}, y$ in units of $k_{2}$, and time in units of $1 / r_{1}$. Then system (2) takes the following form:

$$
\begin{aligned}
& \frac{d x}{d t}=x\left(1-x-\lambda_{1} y\right) \\
& \frac{d y}{d t}=r y\left(1-y-\lambda_{2} x\right),
\end{aligned}
$$

where $\lambda_{i}=k_{i} \zeta_{i}$ for $i=1,2$ and $r=r_{2} / r_{1}$. It is clear that for all parametric values, system (3) has three boundary equilibria $O(0,0), A(1,0), B(0,1)$ and a unique positive equilibrium 
point $C\left(\left(1-\lambda_{1}\right) /\left(1-\lambda_{1} \lambda_{2}\right),\left(1-\lambda_{2}\right) /\left(1-\lambda_{1} \lambda_{2}\right)\right)$ if $\lambda_{1}<$ $1, \lambda_{2}<1, \lambda_{1} \lambda_{2}<1$. According to continuous dynamical systems theory, it is easy to show that equilibrium $O(0,0)$ is a source but never sink and saddle; equilibrium $A(1,0)$ is a sink if $\lambda_{2}>1$ and saddle if $\lambda_{2}<1$, but it is never source; $B(0,1)$ is a sink if $\lambda_{1}>1$ and saddle if $\lambda_{1}<1$, but it is never source, and the unique positive equilibrium point $C\left(\left(1-\lambda_{1}\right) /\left(1-\lambda_{1} \lambda_{2}\right),\left(1-\lambda_{2}\right) /\left(1-\lambda_{1} \lambda_{2}\right)\right)$ is locally asymptotically stable.

A discrete dynamical system is defined as a system whose state evolves over state space in discrete-time steps according to a fixed rule. These systems are represented by a system of difference equations. This is a well-known fact that difference equations existed before differential equations and have played a fundamental role in the development of the latter. During the last fifty years the theory of difference equations received attention of both mathematicians and users of mathematics and developed greatly, because of its internal mathematical beauty and applicability in almost all branches of modern science such as ecology, population dynamics, queuing problems, statistical problems, stochastic time series, number theory, geometry, neural networks, quanta in radiation, genetics in biology, economics, psychology, sociology, physics, engineering, economics, combinatorial analysis, probability theory, electrical networks, and resource management $[6,7]$. Dynamics of a discrete dynamical system is studied by analyzing the behavior of the solution of the system of difference equations representing the system under study. Analyzing the behavior of solutions of a higher-order nonlinear difference equation is very interesting and attracted many researchers in recent times. Behavior of solutions means studying the equilibrium point, boundedness and persistence, existence and uniqueness of positive equilibrium point, local and global stability, periodicity nature of such difference equations or systems of difference equations (see [8-16] and references cited therein).

Discrete dynamical systems described by difference equations are more appropriate for population dynamics as compared to continuous ones. Biologists believe that the equilibrium point and its stability analysis is important to understand the population dynamics $[17,18]$. Therefore, in this paper, we study the behavior of the following discretetime Lotka-Volterra model, which is obtained by discretization of continuous-time model (3) followed by forward Euler's method. Using forward Euler's method, continuoustime model (3) takes the following form:

$$
\begin{aligned}
& \frac{x_{n+1}-x_{n}}{h}=x_{n}-x_{n} x_{n+1}-\lambda_{1} x_{n} y_{n} \\
& \frac{y_{n+1}-y_{n}}{k}=r y_{n}-r y_{n} y_{n+1}-r \lambda_{2} x_{n} y_{n} .
\end{aligned}
$$

After some simplification, the above system becomes

$$
\begin{aligned}
& x_{n+1}=\frac{\alpha_{1} x_{n}-\alpha_{2} x_{n} y_{n}}{1+\alpha_{3} x_{n}} \\
& y_{n+1}=\frac{\alpha_{4} y_{n}-\alpha_{5} x_{n} y_{n}}{1+\alpha_{6} y_{n}},
\end{aligned}
$$

where $\alpha_{1}=1+h, \alpha_{2}=h \lambda_{1}, \alpha_{3}=h, \alpha_{4}=1+$ $k r, \alpha_{5}=k r \lambda_{2}, \alpha_{6}=k r$. It is also noted that the parameters $\alpha_{1}, \alpha_{2}, \alpha_{3}, \alpha_{4}, \alpha_{5}, \alpha_{6}$ and the initial conditions $x_{0}, y_{0}$ are positive real numbers.

The rest of the paper is organized as follows: In Section 2, we study the existence of equilibria of the discrete model. Section 3 deals with the study of local stability of three boundary equilibria and the unique positive equilibrium. Section 4 deals with the study of bifurcations analysis of boundary equilibria and the unique positive equilibrium. Section 5 discusses the boundedness character and the construction of invariant rectangle of the discrete model. Section 6 discusses the global behavior of $O(0,0)$ and the unique positive equilibrium, whereas Section 7 is about numerical simulation to verify the obtained theoretical results. In the last section a brief conclusion is given.

\section{Existence of Equilibria}

In this section, we will study the existence of equilibria of the discrete model (5) in the closed first quadrant $\mathbb{R}^{2}$. The results about the existence of equilibria are summarized as follows.

Lemma 1. Under certain parametric conditions, system (5) has at least three boundary equilibria and one unique positive equilibrium in the closed first quadrant $\mathbb{R}^{2}$. More precisely,

(i) system (5) has a unique boundary equilibrium $O(0,0)$ if $\alpha_{1}<1, \alpha_{4}<1, \alpha_{3} \alpha_{6}<\alpha_{2} \alpha_{5}, \alpha_{6}<\alpha_{2}\left(\alpha_{4}-1\right) /\left(\alpha_{1}-\right.$ 1) and $\alpha_{5}>\alpha_{3}\left(\alpha_{4}-1\right) /\left(\alpha_{1}-1\right)$;

(ii) system (5) has two boundary equilibrium $O(0,0)$, $A\left(\left(\alpha_{1}-1\right) / \alpha_{3}, 0\right)$ if $\alpha_{1}>1, \alpha_{4}<1, \alpha_{3} \alpha_{6}<\alpha_{2} \alpha_{5}$, $\alpha_{6}<\alpha_{2}\left(\alpha_{4}-1\right) /\left(\alpha_{1}-1\right)$ and $\alpha_{5}>\alpha_{3}\left(\alpha_{4}-1\right) /\left(\alpha_{1}-1\right) ;$

(iii) system (5) has three boundary equilibrium $O(0,0)$, $A\left(\left(\alpha_{1}-1\right) / \alpha_{3}, 0\right), B\left(0,\left(\alpha_{4}-1\right) / \alpha_{6}\right)$ if $\alpha_{1}>1, \alpha_{4}>$ $1, \alpha_{3} \alpha_{6}<\alpha_{2} \alpha_{5}, \alpha_{6}<\alpha_{2}\left(\alpha_{4}-1\right) /\left(\alpha_{1}-1\right)$ and $\alpha_{5}>$ $\alpha_{3}\left(\alpha_{4}-1\right) /\left(\alpha_{1}-1\right)$;

(iv) system (5) has three boundary equilibrium $O(0,0)$, $A\left(\left(\alpha_{1}-1\right) / \alpha_{3}, 0\right), B\left(0,\left(\alpha_{4}-1\right) / \alpha_{6}\right)$ and one interior equilibrium $C\left(\left(\left(\alpha_{1}-1\right) \alpha_{6}-\alpha_{2}\left(\alpha_{4}-1\right)\right) /\left(\alpha_{3} \alpha_{6}-\right.\right.$ $\left.\left.\alpha_{2} \alpha_{5}\right),\left(\alpha_{3}\left(\alpha_{4}-1\right)+\alpha_{5}\left(1-\alpha_{1}\right)\right) /\left(\alpha_{3} \alpha_{6}-\alpha_{2} \alpha_{5}\right)\right)$ if $\alpha_{1}>1, \alpha_{4}>1, \alpha_{3} \alpha_{6}>\alpha_{2} \alpha_{5}, \alpha_{6}>\alpha_{2}\left(\alpha_{4}-1\right) /\left(\alpha_{1}-1\right)$ and $\alpha_{5}<\alpha_{3}\left(\alpha_{4}-1\right) /\left(\alpha_{1}-1\right)$. Additionally if $\alpha_{1}>$ $1, \alpha_{4}>1, \alpha_{3} \alpha_{6}>\alpha_{2} \alpha_{5}, \alpha_{6}>\alpha_{2}\left(\alpha_{4}-1\right) /\left(\alpha_{1}-1\right)$ and $\alpha_{5}<\alpha_{3}\left(\alpha_{4}-1\right) /\left(\alpha_{1}-1\right)$, then $C\left(\left(\left(\alpha_{1}-1\right) \alpha_{6}-\alpha_{2}\left(\alpha_{4}-\right.\right.\right.$ $1)) /\left(\alpha_{3} \alpha_{6}-\alpha_{2} \alpha_{5}\right),\left(\alpha_{3}\left(\alpha_{4}-1\right)+\alpha_{5}\left(1-\alpha_{1}\right)\right) /\left(\alpha_{3} \alpha_{6}-\right.$ $\left.\alpha_{2} \alpha_{5}\right)$ ) is the unique positive equilibrium of system (5).

Proof. In order to find equilibria of system (5) in the interior of $\mathbb{R}^{2}$, we need to solve the following algebraic equations:

$$
\begin{aligned}
& x=\frac{\alpha_{1} x-\alpha_{2} x y}{1+\alpha_{3} x} \\
& y=\frac{\alpha_{4} y-\alpha_{5} x y}{1+\alpha_{6} y} .
\end{aligned}
$$

If $x=0, y=0$, then (6) are identically satisfied, and hence for all parametric values $O(0,0)$ is the unique equilibrium 
of this system. If $y=0$ then the second equation of (6) is identically satisfied, and from the first equation we get $x=\left(\alpha_{1}-1\right) / \alpha_{3}$. Thus if $\alpha_{1}>1$, then $A\left(\left(\alpha_{1}-1\right) / \alpha_{3}, 0\right)$ is one boundary equilibrium point of system (5). If $x=0$, then first equation of (6) is identically satisfied, and from the second equation we get $y=\left(\alpha_{4}-1\right) / \alpha_{6}$. Thus if $\alpha_{4}>1$, then $B\left(0,\left(\alpha_{4}-1\right) / \alpha_{6}\right)$ is again a boundary equilibrium of system (5).

On the other hand, we consider the existence of unique positive equilibrium of system (5) in the interior of $\mathbb{R}^{2}$. For this assume that if $x \neq 0$ and $y \neq 0$, then system (6) takes the following form:

$$
\begin{aligned}
& 1=\frac{\alpha_{1}-\alpha_{2} y}{1+\alpha_{3} x} \\
& 1=\frac{\alpha_{4}-\alpha_{5} x}{1+\alpha_{6} y} .
\end{aligned}
$$

Solving system (7) for $x$ and $y$, one gets $(x, y)=\left(\left(\left(\alpha_{1}-1\right) \alpha_{6}-\right.\right.$ $\left.\alpha_{2}\left(\alpha_{4}-1\right)\right) /\left(\alpha_{3} \alpha_{6}-\alpha_{2} \alpha_{5}\right),\left(\alpha_{3}\left(\alpha_{4}-1\right)+\alpha_{5}\left(1-\alpha_{1}\right)\right) /\left(\alpha_{3} \alpha_{6}-\right.$ $\left.\left.\alpha_{2} \alpha_{5}\right)\right)$. Hence if $\alpha_{1}>1, \alpha_{4}>1, \alpha_{3} \alpha_{6}>\alpha_{2} \alpha_{5}, \alpha_{6}>\alpha_{2}\left(\alpha_{4}-\right.$ $1) /\left(\alpha_{1}-1\right)$ and $\alpha_{5}<\alpha_{3}\left(\alpha_{4}-1\right) /\left(\alpha_{1}-1\right)$, then

$$
\begin{gathered}
C\left(\frac{\left(\alpha_{1}-1\right) \alpha_{6}-\alpha_{2}\left(\alpha_{4}-1\right)}{\alpha_{3} \alpha_{6}-\alpha_{2} \alpha_{5}},\right. \\
\left.\frac{\alpha_{3}\left(\alpha_{4}-1\right)+\alpha_{5}\left(1-\alpha_{1}\right)}{\alpha_{3} \alpha_{6}-\alpha_{2} \alpha_{5}}\right)
\end{gathered}
$$

is the unique positive equilibrium of system (5).

Remark 2. The discrete model (5) has three boundary equilibria $O(0,0), A\left(\left(\alpha_{1}-1\right) / \alpha_{3}, 0\right), B\left(0,\left(\alpha_{4}-1\right) / \alpha_{6}\right)$ and one interior equilibrium $C\left(\left(\left(\alpha_{1}-1\right) \alpha_{6}-\alpha_{2}\left(\alpha_{4}-1\right)\right) /\left(\alpha_{3} \alpha_{6}-\right.\right.$ $\left.\left.\alpha_{2} \alpha_{5}\right),\left(\alpha_{3}\left(\alpha_{4}-1\right)+\alpha_{5}\left(1-\alpha_{1}\right)\right) /\left(\alpha_{3} \alpha_{6}-\alpha_{2} \alpha_{5}\right)\right)$ if $\alpha_{1}>$ $1, \alpha_{4}>1, \alpha_{3} \alpha_{6}>\alpha_{2} \alpha_{5}, \alpha_{6}>\alpha_{2}\left(\alpha_{4}-1\right) /\left(\alpha_{1}-1\right)$ and $\alpha_{5}<\alpha_{3}\left(\alpha_{4}-1\right) /\left(\alpha_{1}-1\right)$, where $\alpha_{1}=1+h, \alpha_{2}=h \lambda_{1}, \alpha_{3}=$ $h, \alpha_{4}=1+k r, \alpha_{5}=k r \lambda_{2}, \alpha_{6}=k r$. Now, using the values $\alpha_{i}, i=1, \ldots, 6$, the equilibria of continuous-time model (3), $O(0,0), A(1,0), B(0,1)$, and $C\left(\left(1-\lambda_{1}\right) /\left(1-\lambda_{1} \lambda_{2}\right),(1-\right.$ $\left.\left.\lambda_{2}\right) /\left(1-\lambda_{1} \lambda_{2}\right)\right)$ can be recovered with the same conditions on the parameters $\lambda_{1}<1, \lambda_{2}<1$.

\section{Local Stability}

The Jacobian matrix $J_{(x, y)}$ of linearized system of (5) about equilibrium $(x, y)$ is

$$
J_{(x, y)}=\left(\begin{array}{cc}
\frac{\alpha_{1}-\alpha_{2} y}{\left(1+\alpha_{3} x\right)^{2}} & -\frac{\alpha_{2} x}{1+\alpha_{3} x} \\
-\frac{\alpha_{5} y}{1+\alpha_{6} y} & \frac{\alpha_{4}-\alpha_{5} x}{\left(1+\alpha_{6} y\right)^{2}}
\end{array}\right) .
$$

3.1. Local Stability of Boundary Equilibria. Hereafter we will study the topological classification of the boundary equilibria. The results regarding the local stability of the boundary equilibria are summarized as follows.

Theorem 3. For equilibrium point $\mathrm{O}(0,0)$, the following statements hold: (i) The equilibrium point $\mathrm{O}(0,0)$ of system (5) is a sink if $\alpha_{1}<1$ and $\alpha_{4}<1$

(ii) The equilibrium point $O(0,0)$ of system (5) is a source if $\alpha_{1}>1$ and $\alpha_{4}>1$;

(iii) The equilibrium point $O(0,0)$ of system (5) is a saddle if $\alpha_{1}>1$ and $\alpha_{4}<1$;

(iv) The equilibrium point $O(0,0)$ of system (5) is nonhyperbolic if $\alpha_{1}=1$ or $\alpha_{4}=1$.

Theorem 4. For equilibrium point $A\left(\left(\alpha_{1}-1\right) / \alpha_{3}, 0\right)$, the following statements hold:

(i) The equilibrium $A\left(\left(\alpha_{1}-1\right) / \alpha_{3}, 0\right)$ of system (5) is a sink if $\alpha_{1}>1$ and $\alpha_{5}<\alpha_{3}\left(\alpha_{4}+1\right) /\left(\alpha_{1}-1\right)$;

(ii) The equilibrium $A\left(\left(\alpha_{1}-1\right) / \alpha_{3}, 0\right)$ of system (5) is never source;

(iii) The equilibrium $A\left(\left(\alpha_{1}-1\right) / \alpha_{3}, 0\right)$ of system (5) is a saddle if $\alpha_{1}>1$ and $\alpha_{5}>\alpha_{3}\left(\alpha_{4}+1\right) /\left(\alpha_{1}-1\right)$;

(iv) The equilibrium $A\left(\left(\alpha_{1}-1\right) / \alpha_{3}, 0\right)$ of system (5) is nonhyperbolic if $\alpha_{5}=\alpha_{3}\left(\alpha_{4}+1\right) /\left(\alpha_{1}-1\right)$.

Theorem 5. For equilibrium point $B\left(0,\left(\alpha_{4}-1\right) / \alpha_{6}\right)$, the following statements hold:

(i) The equilibrium $B\left(0,\left(\alpha_{4}-1\right) / \alpha_{6}\right)$ of system (5) is a sink if $\alpha_{4}>1$ and $\alpha_{2}<\left(\alpha_{1}+1\right) \alpha_{6} /\left(\alpha_{4}-1\right)$;

(ii) The equilibrium $B\left(0,\left(\alpha_{4}-1\right) / \alpha_{6}\right)$ of system (5) is never source;

(iii) The equilibrium $B\left(0,\left(\alpha_{4}-1\right) / \alpha_{6}\right)$ of system (5) is a saddle if $\alpha_{4}>1$ and $\alpha_{2}>\left(\alpha_{1}+1\right) \alpha_{6} /\left(\alpha_{4}-1\right)$;

(iv) The equilibrium $B\left(0,\left(\alpha_{4}-1\right) / \alpha_{6}\right)$ of system (5) is nonhyperbolic if $\alpha_{2}=\left(\alpha_{1}+1\right) \alpha_{6} /\left(\alpha_{4}-1\right)$.

Now in the following we will study the local stability of the unique positive equilibrium $C\left(\left(\left(\alpha_{1}-1\right) \alpha_{6}-\alpha_{2}\left(\alpha_{4}-1\right)\right) /\left(\alpha_{3} \alpha_{6}-\right.\right.$ $\left.\left.\alpha_{2} \alpha_{5}\right),\left(\alpha_{3}\left(\alpha_{4}-1\right)+\alpha_{5}\left(1-\alpha_{1}\right)\right) /\left(\alpha_{3} \alpha_{6}-\alpha_{2} \alpha_{5}\right)\right)$ by using Remark 1.3.1 of [7].

\subsection{Local Stability of the Unique Positive Equilibrium}

Theorem 6. For the unique positive equilibrium $C\left(\left(\alpha_{1}-\right.\right.$ 1) $\left.\alpha_{6}-\alpha_{2}\left(\alpha_{4}-1\right)\right) /\left(\alpha_{3} \alpha_{6}-\alpha_{2} \alpha_{5}\right),\left(\alpha_{3}\left(\alpha_{4}-1\right)+\alpha_{5}\left(1-\alpha_{1}\right)\right) /\left(\alpha_{3} \alpha_{6}-\right.$ $\left.\left.\alpha_{2} \alpha_{5}\right)\right)$ of system (5), the following statements hold:

(i) The unique positive equilibrium point $C\left(\left(\left(\alpha_{1}-1\right) \alpha_{6}-\right.\right.$ $\left.\alpha_{2}\left(\alpha_{4}-1\right)\right) /\left(\alpha_{3} \alpha_{6}-\alpha_{2} \alpha_{5}\right),\left(\alpha_{3}\left(\alpha_{4}-1\right)+\alpha_{5}(1-\right.$ $\left.\left.\left.\alpha_{1}\right)\right) /\left(\alpha_{3} \alpha_{6}-\alpha_{2} \alpha_{5}\right)\right)$ of system (5) is locally asymptotically stable if

$$
\begin{aligned}
\Theta< & \left(\alpha_{2} \alpha_{3}-\alpha_{2} \alpha_{3} \alpha_{4}-\alpha_{2} \alpha_{5}+\alpha_{1} \alpha_{3} \alpha_{6}\right) \\
& \cdot\left(-\alpha_{2} \alpha_{5}+\alpha_{3} \alpha_{4} \alpha_{6}+\alpha_{5} \alpha_{6}-\alpha_{1} \alpha_{5} \alpha_{6}\right) ;
\end{aligned}
$$


(ii) The unique positive equilibrium point $C\left(\left(\left(\alpha_{1}-1\right) \alpha_{6}-\right.\right.$ $\left.\alpha_{2}\left(\alpha_{4}-1\right)\right) /\left(\alpha_{3} \alpha_{6}-\alpha_{2} \alpha_{5}\right),\left(\alpha_{3}\left(\alpha_{4}-1\right)+\alpha_{5}\left(1-\alpha_{1}\right)\right) /$ $\left.\left(\alpha_{3} \alpha_{6}-\alpha_{2} \alpha_{5}\right)\right)$ of system (5) is unstable if

$$
\begin{aligned}
\Theta> & \left(\alpha_{2} \alpha_{3}-\alpha_{2} \alpha_{3} \alpha_{4}-\alpha_{2} \alpha_{5}+\alpha_{1} \alpha_{3} \alpha_{6}\right) \\
& \cdot\left(-\alpha_{2} \alpha_{5}+\alpha_{3} \alpha_{4} \alpha_{6}+\alpha_{5} \alpha_{6}-\alpha_{1} \alpha_{5} \alpha_{6}\right),
\end{aligned}
$$

where

$$
\begin{aligned}
\Theta= & -2 \alpha_{2} \alpha_{3} \alpha_{6} \alpha_{5}+\alpha_{3}{ }^{2} \alpha_{6}{ }^{2}\left(\alpha_{1}+\alpha_{4}+1\right) \\
& +\alpha_{6}{ }^{2} \alpha_{3} \alpha_{5}\left(1-\alpha_{1}\right)+\alpha_{3}{ }^{2} \alpha_{6} \alpha_{2}\left(1-\alpha_{4}\right)
\end{aligned}
$$

$$
\begin{aligned}
& +\alpha_{2}^{2} \alpha_{5}^{2}\left(\left(\alpha_{1}+1\right) \alpha_{4}+\alpha_{1}-2\right) \\
& +\alpha_{5}^{2} \alpha_{2} \alpha_{6}\left(2+\alpha_{1}\left(\alpha_{1}+1\right)\right) \\
& +\alpha_{2}{ }^{2} \alpha_{3} \alpha_{5}\left(2+\alpha_{4}\left(\alpha_{4}+1\right)\right) \\
& +\alpha_{2} \alpha_{3} \alpha_{6} \alpha_{5}\left(3+\left(\alpha_{1}+2\right) \alpha_{4}+2 \alpha_{1}\right) .
\end{aligned}
$$

Proof. (i) The Jacobian matrix $J_{C\left(\left(\left(\alpha_{1}-1\right) \alpha_{6}-\alpha_{2}\left(\alpha_{4}-1\right)\right) /\left(\alpha_{3} \alpha_{6}-\alpha_{2} \alpha_{5}\right),\left(\alpha_{3}\left(\alpha_{4}-1\right)+\alpha_{5}\left(1-\alpha_{1}\right)\right) /\left(\alpha_{3} \alpha_{6}-\alpha_{2} \alpha_{5}\right)\right) \quad \text { of }}$ linearized system of (5) about $C\left(\left(\left(\alpha_{1}-1\right) \alpha_{6}-\alpha_{2}\left(\alpha_{4}-1\right)\right) /\right.$ $\left.\left(\alpha_{3} \alpha_{6}-\alpha_{2} \alpha_{5}\right),\left(\alpha_{3}\left(\alpha_{4}-1\right)+\alpha_{5}\left(1-\alpha_{1}\right)\right) /\left(\alpha_{3} \alpha_{6}-\alpha_{2} \alpha_{5}\right)\right)$ is

$J_{C\left(\left(\left(\alpha_{1}-1\right) \alpha_{6}-\alpha_{2}\left(\alpha_{4}-1\right)\right) /\left(\alpha_{3} \alpha_{6}-\alpha_{2} \alpha_{5}\right),\left(\alpha_{3}\left(\alpha_{4}-1\right)+\alpha_{5}\left(1-\alpha_{1}\right)\right) /\left(\alpha_{3} \alpha_{6}-\alpha_{2} \alpha_{5}\right)\right)}$

$$
=\left(\begin{array}{cc}
\frac{\alpha_{3} \alpha_{6}-\alpha_{2} \alpha_{5}}{\alpha_{1} \alpha_{3} \alpha_{6}-\alpha_{2} \alpha_{5}-\alpha_{2} \alpha_{3} \alpha_{4}+\alpha_{2} \alpha_{3}} & -\frac{\alpha_{2} \alpha_{6}\left(\alpha_{1}-1\right)-\alpha_{2}^{2}\left(\alpha_{4}-1\right)}{\alpha_{1} \alpha_{3} \alpha_{6}-\alpha_{2} \alpha_{5}-\alpha_{2} \alpha_{3} \alpha_{4}+\alpha_{2} \alpha_{3}} \\
-\frac{\alpha_{3} \alpha_{5}\left(\alpha_{4}-1\right)-\alpha_{5}^{2}\left(\alpha_{1}-1\right)}{\alpha_{3} \alpha_{6} \alpha_{4}-\alpha_{2} \alpha_{5}-\alpha_{6} \alpha_{5} \alpha_{1}+\alpha_{6} \alpha_{5}} & \frac{\alpha_{3} \alpha_{6}-\alpha_{2} \alpha_{5}}{\alpha_{3} \alpha_{6} \alpha_{4}-\alpha_{2} \alpha_{5}-\alpha_{6} \alpha_{5} \alpha_{1}+\alpha_{6} \alpha_{5}}
\end{array}\right) .
$$

The characteristic polynomial of is

$J_{C\left(\left(\left(\alpha_{1}-1\right) \alpha_{6}-\alpha_{2}\left(\alpha_{4}-1\right)\right) /\left(\alpha_{3} \alpha_{6}-\alpha_{2} \alpha_{5}\right),\left(\alpha_{3}\left(\alpha_{4}-1\right)+\alpha_{5}\left(1-\alpha_{1}\right)\right) /\left(\alpha_{3} \alpha_{6}-\alpha_{2} \alpha_{5}\right)\right)}$ about

$$
\begin{gathered}
C\left(\frac{\left(\alpha_{1}-1\right) \alpha_{6}-\alpha_{2}\left(\alpha_{4}-1\right)}{\alpha_{3} \alpha_{6}-\alpha_{2} \alpha_{5}},\right. \\
\left.\frac{\alpha_{3}\left(\alpha_{4}-1\right)+\alpha_{5}\left(1-\alpha_{1}\right)}{\alpha_{3} \alpha_{6}-\alpha_{2} \alpha_{5}}\right)
\end{gathered}
$$

$$
P(\lambda)=\lambda^{2}-\Omega_{1} \lambda+\Omega_{2}
$$

where

$$
\begin{aligned}
& \Omega_{1}=A+B-C-D, \\
& \Omega_{2}=E-F+G+H+I-J-K+L+M-N+O-P+Q+R, \\
& A=\frac{\alpha_{3} \alpha_{6}}{\alpha_{2} \alpha_{3}-\alpha_{2} \alpha_{3} \alpha_{4}-\alpha_{2} \alpha_{5}+\alpha_{1} \alpha_{3} \alpha_{6}}, \\
& B=\frac{\alpha_{3} \alpha_{6}}{-\alpha_{2} \alpha_{5}+\alpha_{3} \alpha_{4} \alpha_{6}+\alpha_{5} \alpha_{6}-\alpha_{1} \alpha_{5} \alpha_{6}}, \\
& C=\frac{\alpha_{2} \alpha_{5}}{\alpha_{2} \alpha_{3}-\alpha_{2} \alpha_{3} \alpha_{4}-\alpha_{2} \alpha_{5}+\alpha_{1} \alpha_{3} \alpha_{6}}, \\
& D=\frac{\alpha_{2} \alpha_{5}}{-\alpha_{2} \alpha_{5}+\alpha_{3} \alpha_{4} \alpha_{6}+\alpha_{5} \alpha_{6}-\alpha_{1} \alpha_{5} \alpha_{6}}, \\
& E=\frac{\alpha_{2}{ }^{2} \alpha_{3} \alpha_{5}}{\left(\alpha_{2} \alpha_{3}-\alpha_{2} \alpha_{3} \alpha_{4}-\alpha_{2} \alpha_{5}+\alpha_{1} \alpha_{3} \alpha_{6}\right)\left(-\alpha_{2} \alpha_{5}+\alpha_{3} \alpha_{4} \alpha_{6}+\alpha_{5} \alpha_{6}-\alpha_{1} \alpha_{5} \alpha_{6}\right)}, \\
& F=\frac{2 \alpha_{2}{ }^{2} \alpha_{3} \alpha_{4} \alpha_{5}}{\left(\alpha_{2} \alpha_{3}-\alpha_{2} \alpha_{3} \alpha_{4}-\alpha_{2} \alpha_{5}+\alpha_{1} \alpha_{3} \alpha_{6}\right)\left(-\alpha_{2} \alpha_{5}+\alpha_{3} \alpha_{4} \alpha_{6}+\alpha_{5} \alpha_{6}-\alpha_{1} \alpha_{5} \alpha_{6}\right)}, \\
& G=\frac{\alpha_{2}{ }^{2} \alpha_{3} \alpha_{4}{ }^{2} \alpha_{5}}{\left(\alpha_{2} \alpha_{3}-\alpha_{2} \alpha_{3} \alpha_{4}-\alpha_{2} \alpha_{5}+\alpha_{1} \alpha_{3} \alpha_{6}\right)\left(-\alpha_{2} \alpha_{5}+\alpha_{3} \alpha_{4} \alpha_{6}+\alpha_{5} \alpha_{6}-\alpha_{1} \alpha_{5} \alpha_{6}\right)},
\end{aligned}
$$




$$
\begin{aligned}
& H=\frac{\alpha_{1} \alpha_{2}{ }^{2} \alpha_{5}^{2}}{\left(\alpha_{2} \alpha_{3}-\alpha_{2} \alpha_{3} \alpha_{4}-\alpha_{2} \alpha_{5}+\alpha_{1} \alpha_{3} \alpha_{6}\right)\left(-\alpha_{2} \alpha_{5}+\alpha_{3} \alpha_{4} \alpha_{6}+\alpha_{5} \alpha_{6}-\alpha_{1} \alpha_{5} \alpha_{6}\right)}, \\
& I=\frac{\alpha_{2}^{2} \alpha_{4} \alpha_{5}^{2}}{\left(\alpha_{2} \alpha_{3}-\alpha_{2} \alpha_{3} \alpha_{4}-\alpha_{2} \alpha_{5}+\alpha_{1} \alpha_{3} \alpha_{6}\right)\left(-\alpha_{2} \alpha_{5}+\alpha_{3} \alpha_{4} \alpha_{6}+\alpha_{5} \alpha_{6}-\alpha_{1}-\alpha_{1} \alpha_{5} \alpha_{6}\right)}, \\
& J=\frac{\alpha_{1} \alpha_{2}^{2} \alpha_{4} \alpha_{5}^{2}}{\left(\alpha_{2} \alpha_{3}-\alpha_{2} \alpha_{3} \alpha_{4}-\alpha_{2} \alpha_{5}+\alpha_{1} \alpha_{3} \alpha_{6}\right)\left(-\alpha_{2} \alpha_{5}+\alpha_{3} \alpha_{4} \alpha_{6}+\alpha_{5} \alpha_{6}-\alpha_{1} \alpha_{5} \alpha_{6}\right)}, \\
& K=\frac{3 \alpha_{2} \alpha_{3} \alpha_{5} \alpha_{6}}{\left(\alpha_{2} \alpha_{3}-\alpha_{2} \alpha_{3} \alpha_{4}-\alpha_{2} \alpha_{5}+\alpha_{1} \alpha_{3} \alpha_{6}\right)\left(-\alpha_{2} \alpha_{5}+\alpha_{3} \alpha_{4} \alpha_{6}+\alpha_{5} \alpha_{6}-\alpha_{1} \alpha_{5} \alpha_{6}\right)}, \\
& L=\frac{\alpha_{1} \alpha_{2} \alpha_{3} \alpha_{5} \alpha_{6}}{\left(\alpha_{2} \alpha_{3}-\alpha_{2} \alpha_{3} \alpha_{4}-\alpha_{2} \alpha_{5}+\alpha_{1} \alpha_{3} \alpha_{6}\right)\left(-\alpha_{2} \alpha_{5}+\alpha_{3} \alpha_{4} \alpha_{6}+\alpha_{5} \alpha_{6}-\alpha_{1} \alpha_{5} \alpha_{6}\right)}, \\
& M=\frac{\alpha_{2} \alpha_{3} \alpha_{4} \alpha_{5} \alpha_{6}}{\left(\alpha_{2} \alpha_{3}-\alpha_{2} \alpha_{3} \alpha_{4}-\alpha_{2} \alpha_{5}+\alpha_{1} \alpha_{3} \alpha_{6}\right)\left(-\alpha_{2} \alpha_{5}+\alpha_{3} \alpha_{4} \alpha_{6}+\alpha_{5} \alpha_{6}-\alpha_{1} \alpha_{5} \alpha_{6}\right)}, \\
& N=\frac{\alpha_{1} \alpha_{2} \alpha_{3} \alpha_{4} \alpha_{5} \alpha_{6}}{\left(\alpha_{2} \alpha_{3}-\alpha_{2} \alpha_{3} \alpha_{4}-\alpha_{2} \alpha_{5}+\alpha_{1} \alpha_{3} \alpha_{6}\right)\left(-\alpha_{2} \alpha_{5}+\alpha_{3} \alpha_{4} \alpha_{6}+\alpha_{5} \alpha_{6}-\alpha_{1} \alpha_{5} \alpha_{6}\right)}, \\
& O=\frac{\alpha_{2} \alpha_{5}^{2} \alpha_{6}}{\left(\alpha_{2} \alpha_{3}-\alpha_{2} \alpha_{3} \alpha_{4}-\alpha_{2} \alpha_{5}+\alpha_{1} \alpha_{3} \alpha_{6}\right)\left(-\alpha_{2} \alpha_{5}+\alpha_{3} \alpha_{4} \alpha_{6}+\alpha_{5} \alpha_{6}-\alpha_{1} \alpha_{5} \alpha_{6}\right)}, \\
& P=\frac{2 \alpha_{1} \alpha_{2} \alpha_{5}^{2} \alpha_{6}}{\left(\alpha_{2} \alpha_{3}-\alpha_{2} \alpha_{3} \alpha_{4}-\alpha_{2} \alpha_{5}+\alpha_{1} \alpha_{3} \alpha_{6}\right)\left(-\alpha_{2} \alpha_{5}+\alpha_{3} \alpha_{4} \alpha_{6}+\alpha_{5} \alpha_{6}-\alpha_{1} \alpha_{5} \alpha_{6}\right)}, \\
& Q=\frac{\alpha_{1}^{2} \alpha_{2} \alpha_{5}^{2} \alpha_{6}}{\left(\alpha_{2} \alpha_{3}-\alpha_{2} \alpha_{3} \alpha_{4}-\alpha_{2} \alpha_{5}+\alpha_{1} \alpha_{3} \alpha_{6}\right)\left(-\alpha_{2} \alpha_{5}+\alpha_{3} \alpha_{4} \alpha_{6}+\alpha_{5} \alpha_{6}-\alpha_{1} \alpha_{5} \alpha_{6}\right)}, \\
& R=\frac{\alpha_{3}{ }^{2} \alpha_{6}{ }^{2}}{\left(\alpha_{2} \alpha_{3}-\alpha_{2} \alpha_{3} \alpha_{4}-\alpha_{2} \alpha_{5}+\alpha_{1} \alpha_{3} \alpha_{6}\right)\left(-\alpha_{2} \alpha_{5}+\alpha_{3} \alpha_{4} \alpha_{6}+\alpha_{5} \alpha_{6}-\alpha_{1} \alpha_{5} \alpha_{6}\right)} .
\end{aligned}
$$

Assume that $\Theta<\left(\alpha_{2} \alpha_{3}-\alpha_{2} \alpha_{3} \alpha_{4}-\alpha_{2} \alpha_{5}+\alpha_{1} \alpha_{3} \alpha_{6}\right)\left(-\alpha_{2} \alpha_{5}+\right.$ $\left.\alpha_{3} \alpha_{4} \alpha_{6}+\alpha_{5} \alpha_{6}-\alpha_{1} \alpha_{5} \alpha_{6}\right)$, and using Remark 1.3.1 of [7] one gets

$$
\begin{aligned}
\left|\Omega_{1}\right|+\left|\Omega_{2}\right| & \leq A+B+C+D+E+F+G+H+I+J+K+L+M+N+O+P+Q+R \\
& <\frac{\Theta}{\left(\alpha_{2} \alpha_{3}-\alpha_{2} \alpha_{3} \alpha_{4}-\alpha_{2} \alpha_{5}+\alpha_{1} \alpha_{3} \alpha_{6}\right)\left(-\alpha_{2} \alpha_{5}+\alpha_{3} \alpha_{4} \alpha_{6}+\alpha_{5} \alpha_{6}-\alpha_{1} \alpha_{5} \alpha_{6}\right)}<1 .
\end{aligned}
$$

Therefore $C\left(\left(\left(\alpha_{1}-1\right) \alpha_{6}-\alpha_{2}\left(\alpha_{4}-1\right)\right) /\left(\alpha_{3} \alpha_{6}-\alpha_{2} \alpha_{5}\right),\left(\alpha_{3}\left(\alpha_{4}-\right.\right.\right.$ 1) $\left.\left.+\alpha_{5}\left(1-\alpha_{1}\right)\right) /\left(\alpha_{3} \alpha_{6}-\alpha_{2} \alpha_{5}\right)\right)$ of system (5) is locally asymptotically stable.

(ii) Similarly it is easy to show that $C\left(\left(\left(\alpha_{1}-1\right) \alpha_{6}-\alpha_{2}\left(\alpha_{4}-\right.\right.\right.$ $\left.1)) /\left(\alpha_{3} \alpha_{6}-\alpha_{2} \alpha_{5}\right),\left(\alpha_{3}\left(\alpha_{4}-1\right)+\alpha_{5}\left(1-\alpha_{1}\right)\right) /\left(\alpha_{3} \alpha_{6}-\alpha_{2} \alpha_{5}\right)\right)$ of system (5) is unstable if $\Theta>\left(\alpha_{2} \alpha_{3}-\alpha_{2} \alpha_{3} \alpha_{4}-\alpha_{2} \alpha_{5}+\right.$ $\left.\alpha_{1} \alpha_{3} \alpha_{6}\right)\left(-\alpha_{2} \alpha_{5}+\alpha_{3} \alpha_{4} \alpha_{6}+\alpha_{5} \alpha_{6}-\alpha_{1} \alpha_{5} \alpha_{6}\right)$.

Hereafter we will compute the necessary and sufficient condition(s) for the unique positive equilibrium $C\left(\left(\alpha_{1}-\right.\right.$
1) $\left.\alpha_{6}-\alpha_{2}\left(\alpha_{4}-1\right)\right) /\left(\alpha_{3} \alpha_{6}-\alpha_{2} \alpha_{5}\right),\left(\alpha_{3}\left(\alpha_{4}-1\right)+\alpha_{5}\left(1-\alpha_{1}\right)\right) /$ $\left.\left(\alpha_{3} \alpha_{6}-\alpha_{2} \alpha_{5}\right)\right)$ of system (5) to be locally asymptotically stable, repeller, saddle, and nonhyperbolic, respectively.

Theorem 7. For equilibrium $C\left(\left(\left(\alpha_{1}-1\right) \alpha_{6}-\alpha_{2}\left(\alpha_{4}-1\right)\right) /\left(\alpha_{3} \alpha_{6}-\right.\right.$ $\left.\left.\alpha_{2} \alpha_{5}\right),\left(\alpha_{3}\left(\alpha_{4}-1\right)+\alpha_{5}\left(1-\alpha_{1}\right)\right) /\left(\alpha_{3} \alpha_{6}-\alpha_{2} \alpha_{5}\right)\right)$ of system (5), the following statements hold:

(i) Equilibrium $C\left(\left(\left(\alpha_{1}-1\right) \alpha_{6}-\alpha_{2}\left(\alpha_{4}-1\right)\right) /\left(\alpha_{3} \alpha_{6}-\right.\right.$ $\left.\left.\alpha_{2} \alpha_{5}\right),\left(\alpha_{3}\left(\alpha_{4}-1\right)+\alpha_{5}\left(1-\alpha_{1}\right)\right) /\left(\alpha_{3} \alpha_{6}-\alpha_{2} \alpha_{5}\right)\right)$ of system (5) is locally asymptotically stable if and only if 


$$
\begin{aligned}
& \left|\frac{\left(\alpha_{3} \alpha_{6}-\alpha_{2} \alpha_{5}\right)\left(\alpha_{3}\left(\alpha_{6}\left(\alpha_{1}+\alpha_{4}\right)+\alpha_{2}\left(1-\alpha_{4}\right)\right)+\alpha_{5}\left(\alpha_{6}\left(1-\alpha_{1}\right)-2 \alpha_{2}\right)\right)}{\left(\alpha_{1} \alpha_{3} \alpha_{6}-\alpha_{2} \alpha_{5}+\alpha_{2} \alpha_{3}\left(\alpha_{4}-1\right)\right)\left(\alpha_{3} \alpha_{6} \alpha_{4}-\alpha_{2} \alpha_{5}+\alpha_{6} \alpha_{5}\left(1-\alpha_{1}\right)\right)}\right| \\
& <1-\frac{\left(\alpha_{3} \alpha_{6}-\alpha_{2} \alpha_{5}\right)^{2}-\left(\alpha_{2} \alpha_{6}\left(\alpha_{1}-1\right)-\alpha_{2}^{2}\left(\alpha_{4}-1\right)\right)\left(\alpha_{3} \alpha_{5}\left(\alpha_{4}-1\right)-\alpha_{5}^{2}\left(\alpha_{1}-1\right)\right)}{\left(\alpha_{1} \alpha_{3} \alpha_{6}-\alpha_{2} \alpha_{5}+\alpha_{2} \alpha_{3}\left(\alpha_{4}-1\right)\right)\left(\alpha_{3} \alpha_{6} \alpha_{4}-\alpha_{2} \alpha_{5}+\alpha_{6} \alpha_{5}\left(1-\alpha_{1}\right)\right)}<2 .
\end{aligned}
$$

(ii) Equilibrium $C\left(\left(\left(\alpha_{1}-1\right) \alpha_{6}-\alpha_{2}\left(\alpha_{4}-1\right)\right) /\left(\alpha_{3} \alpha_{6}-\alpha_{2} \alpha_{5}\right)\right.$, $\left.\left(\alpha_{3}\left(\alpha_{4}-1\right)+\alpha_{5}\left(1-\alpha_{1}\right)\right) /\left(\alpha_{3} \alpha_{6}-\alpha_{2} \alpha_{5}\right)\right)$ of system (5) is a repeller if and only if

$$
\begin{aligned}
& \left|\frac{\left(\alpha_{3} \alpha_{6}-\alpha_{2} \alpha_{5}\right)^{2}-\left(\alpha_{2} \alpha_{6}\left(\alpha_{1}-1\right)-\alpha_{2}^{2}\left(\alpha_{4}-1\right)\right)\left(\alpha_{3} \alpha_{5}\left(\alpha_{4}-1\right)-\alpha_{5}^{2}\left(\alpha_{1}-1\right)\right)}{\left(\alpha_{1} \alpha_{3} \alpha_{6}-\alpha_{2} \alpha_{5}+\alpha_{2} \alpha_{3}\left(\alpha_{4}-1\right)\right)\left(\alpha_{3} \alpha_{6} \alpha_{4}-\alpha_{2} \alpha_{5}+\alpha_{6} \alpha_{5}\left(1-\alpha_{1}\right)\right)}\right|>1, \\
& \left|\frac{\left(\alpha_{3} \alpha_{6}-\alpha_{2} \alpha_{5}\right)\left(\alpha_{3}\left(\alpha_{6}\left(\alpha_{1}+\alpha_{4}\right)+\alpha_{2}\left(1-\alpha_{4}\right)\right)+\alpha_{5}\left(\alpha_{6}\left(1-\alpha_{1}\right)-2 \alpha_{2}\right)\right)}{\left(\alpha_{1} \alpha_{3} \alpha_{6}-\alpha_{2} \alpha_{5}+\alpha_{2} \alpha_{3}\left(\alpha_{4}-1\right)\right)\left(\alpha_{3} \alpha_{6} \alpha_{4}-\alpha_{2} \alpha_{5}+\alpha_{6} \alpha_{5}\left(1-\alpha_{1}\right)\right)}\right| \\
& <\left|1-\frac{\left(\alpha_{3} \alpha_{6}-\alpha_{2} \alpha_{5}\right)^{2}-\left(\alpha_{2} \alpha_{6}\left(\alpha_{1}-1\right)-\alpha_{2}^{2}\left(\alpha_{4}-1\right)\right)\left(\alpha_{3} \alpha_{5}\left(\alpha_{4}-1\right)-\alpha_{5}^{2}\left(\alpha_{1}-1\right)\right)}{\left(\alpha_{1} \alpha_{3} \alpha_{6}-\alpha_{2} \alpha_{5}+\alpha_{2} \alpha_{3}\left(\alpha_{4}-1\right)\right)\left(\alpha_{3} \alpha_{6} \alpha_{4}-\alpha_{2} \alpha_{5}+\alpha_{6} \alpha_{5}\left(1-\alpha_{1}\right)\right)}\right| .
\end{aligned}
$$

(iii) Equilibrium $C\left(\left(\left(\alpha_{1}-1\right) \alpha_{6}-\alpha_{2}\left(\alpha_{4}-1\right)\right) /\left(\alpha_{3} \alpha_{6}-\alpha_{2} \alpha_{5}\right)\right.$, $\left.\left(\alpha_{3}\left(\alpha_{4}-1\right)+\alpha_{5}\left(1-\alpha_{1}\right)\right) /\left(\alpha_{3} \alpha_{6}-\alpha_{2} \alpha_{5}\right)\right)$ of system (5) is a saddle if and only if

$$
\begin{aligned}
& \left(\frac{\left(\alpha_{3} \alpha_{6}-\alpha_{2} \alpha_{5}\right)\left(\alpha_{3}\left(\alpha_{6}\left(\alpha_{1}+\alpha_{4}\right)+\alpha_{2}\left(1-\alpha_{4}\right)\right)+\alpha_{5}\left(\alpha_{6}\left(1-\alpha_{1}\right)-2 \alpha_{2}\right)\right)}{\left(\alpha_{1} \alpha_{3} \alpha_{6}-\alpha_{2} \alpha_{5}+\alpha_{2} \alpha_{3}\left(\alpha_{4}-1\right)\right)\left(\alpha_{3} \alpha_{6} \alpha_{4}-\alpha_{2} \alpha_{5}+\alpha_{6} \alpha_{5}\left(1-\alpha_{1}\right)\right)}\right)^{2} \\
& \quad+4\left(1-\frac{\left(\alpha_{3} \alpha_{6}-\alpha_{2} \alpha_{5}\right)^{2}-\left(\alpha_{2} \alpha_{6}\left(\alpha_{1}-1\right)-\alpha_{2}^{2}\left(\alpha_{4}-1\right)\right)\left(\alpha_{3} \alpha_{5}\left(\alpha_{4}-1\right)-\alpha_{5}^{2}\left(\alpha_{1}-1\right)\right)}{\left(\alpha_{1} \alpha_{3} \alpha_{6}-\alpha_{2} \alpha_{5}+\alpha_{2} \alpha_{3}\left(\alpha_{4}-1\right)\right)\left(\alpha_{3} \alpha_{6} \alpha_{4}-\alpha_{2} \alpha_{5}+\alpha_{6} \alpha_{5}\left(1-\alpha_{1}\right)\right)}\right)>0, \\
& \left|\frac{\left(\alpha_{3} \alpha_{6}-\alpha_{2} \alpha_{5}\right)\left(\alpha_{3}\left(\alpha_{6}\left(\alpha_{1}+\alpha_{4}\right)+\alpha_{2}\left(1-\alpha_{4}\right)\right)+\alpha_{5}\left(\alpha_{6}\left(1-\alpha_{1}\right)-2 \alpha_{2}\right)\right)}{\left(\alpha_{1} \alpha_{3} \alpha_{6}-\alpha_{2} \alpha_{5}+\alpha_{2} \alpha_{3}\left(\alpha_{4}-1\right)\right)\left(\alpha_{3} \alpha_{6} \alpha_{4}-\alpha_{2} \alpha_{5}+\alpha_{6} \alpha_{5}\left(1-\alpha_{1}\right)\right)}\right| \\
& >\left|1-\frac{\left(\alpha_{3} \alpha_{6}-\alpha_{2} \alpha_{5}\right)^{2}-\left(\alpha_{2} \alpha_{6}\left(\alpha_{1}-1\right)-\alpha_{2}^{2}\left(\alpha_{4}-1\right)\right)\left(\alpha_{3} \alpha_{5}\left(\alpha_{4}-1\right)-\alpha_{5}^{2}\left(\alpha_{1}-1\right)\right)}{\left(\alpha_{1} \alpha_{3} \alpha_{6}-\alpha_{2} \alpha_{5}+\alpha_{2} \alpha_{3}\left(\alpha_{4}-1\right)\right)\left(\alpha_{3} \alpha_{6} \alpha_{4}-\alpha_{2} \alpha_{5}+\alpha_{6} \alpha_{5}\left(1-\alpha_{1}\right)\right)}\right| .
\end{aligned}
$$

(iv) Equilibrium $C\left(\left(\left(\alpha_{1}-1\right) \alpha_{6}-\alpha_{2}\left(\alpha_{4}-1\right)\right) /\left(\alpha_{3} \alpha_{6}-\alpha_{2} \alpha_{5}\right)\right.$, $\left.\left(\alpha_{3}\left(\alpha_{4}-1\right)+\alpha_{5}\left(1-\alpha_{1}\right)\right) /\left(\alpha_{3} \alpha_{6}-\alpha_{2} \alpha_{5}\right)\right)$ of system (5) is nonhyperbolic if and only if

$$
\begin{aligned}
& \left|\frac{\left(\alpha_{3} \alpha_{6}-\alpha_{2} \alpha_{5}\right)\left(\alpha_{3}\left(\alpha_{6}\left(\alpha_{1}+\alpha_{4}\right)+\alpha_{2}\left(1-\alpha_{4}\right)\right)+\alpha_{5}\left(\alpha_{6}\left(1-\alpha_{1}\right)-2 \alpha_{2}\right)\right)}{\left(\alpha_{1} \alpha_{3} \alpha_{6}-\alpha_{2} \alpha_{5}+\alpha_{2} \alpha_{3}\left(\alpha_{4}-1\right)\right)\left(\alpha_{3} \alpha_{6} \alpha_{4}-\alpha_{2} \alpha_{5}+\alpha_{6} \alpha_{5}\left(1-\alpha_{1}\right)\right)}\right| \\
& \quad=\left|1-\frac{\left(\alpha_{3} \alpha_{6}-\alpha_{2} \alpha_{5}\right)^{2}-\left(\alpha_{2} \alpha_{6}\left(\alpha_{1}-1\right)-\alpha_{2}^{2}\left(\alpha_{4}-1\right)\right)\left(\alpha_{3} \alpha_{5}\left(\alpha_{4}-1\right)-\alpha_{5}^{2}\left(\alpha_{1}-1\right)\right)}{\left(\alpha_{1} \alpha_{3} \alpha_{6}-\alpha_{2} \alpha_{5}+\alpha_{2} \alpha_{3}\left(\alpha_{4}-1\right)\right)\left(\alpha_{3} \alpha_{6} \alpha_{4}-\alpha_{2} \alpha_{5}+\alpha_{6} \alpha_{5}\left(1-\alpha_{1}\right)\right)}\right| .
\end{aligned}
$$


Proof. (i) More precisely the characteristic equation of $J_{C\left(\left(\left(\alpha_{1}-1\right) \alpha_{6}-\alpha_{2}\left(\alpha_{4}-1\right)\right) /\left(\alpha_{3} \alpha_{6}-\alpha_{2} \alpha_{5}\right),\left(\alpha_{3}\left(\alpha_{4}-1\right)+\alpha_{5}\left(1-\alpha_{1}\right)\right) /\left(\alpha_{3} \alpha_{6}-\alpha_{2} \alpha_{5}\right)\right)}$ about

$$
\begin{gathered}
C\left(\frac{\left(\alpha_{1}-1\right) \alpha_{6}-\alpha_{2}\left(\alpha_{4}-1\right)}{\alpha_{3} \alpha_{6}-\alpha_{2} \alpha_{5}},\right. \\
\left.\frac{\alpha_{3}\left(\alpha_{4}-1\right)+\alpha_{5}\left(1-\alpha_{1}\right)}{\alpha_{3} \alpha_{6}-\alpha_{2} \alpha_{5}}\right)
\end{gathered}
$$

$$
\begin{aligned}
& p=\frac{\left(\alpha_{3} \alpha_{6}-\alpha_{2} \alpha_{5}\right)\left(\alpha_{3}\left(\alpha_{6}\left(\alpha_{1}+\alpha_{4}\right)+\alpha_{2}\left(1-\alpha_{4}\right)\right)+\alpha_{5}\left(\alpha_{6}\left(1-\alpha_{1}\right)-2 \alpha_{2}\right)\right)}{\left(\alpha_{1} \alpha_{3} \alpha_{6}-\alpha_{2} \alpha_{5}+\alpha_{2} \alpha_{3}\left(\alpha_{4}-1\right)\right)\left(\alpha_{3} \alpha_{6} \alpha_{4}-\alpha_{2} \alpha_{5}+\alpha_{6} \alpha_{5}\left(1-\alpha_{1}\right)\right)} \\
& q=\frac{\left(\alpha_{3} \alpha_{6}-\alpha_{2} \alpha_{5}\right)^{2}-\left(\alpha_{2} \alpha_{6}\left(\alpha_{1}-1\right)-\alpha_{2}^{2}\left(\alpha_{4}-1\right)\right)\left(\alpha_{3} \alpha_{5}\left(\alpha_{4}-1\right)-\alpha_{5}^{2}\left(\alpha_{1}-1\right)\right)}{\left(\alpha_{1} \alpha_{3} \alpha_{6}-\alpha_{2} \alpha_{5}+\alpha_{2} \alpha_{3}\left(\alpha_{4}-1\right)\right)\left(\alpha_{3} \alpha_{6} \alpha_{4}-\alpha_{2} \alpha_{5}+\alpha_{6} \alpha_{5}\left(1-\alpha_{1}\right)\right)} .
\end{aligned}
$$

Then, it follows from Theorem 1.1.1 of [12] that the unique positive equilibrium of system (1) is locally asymptotically stable if and only if

$$
\begin{aligned}
& C\left(\frac{\left(\alpha_{1}-1\right) \alpha_{6}-\alpha_{2}\left(\alpha_{4}-1\right)}{\alpha_{3} \alpha_{6}-\alpha_{2} \alpha_{5}},\right. \\
& \left.\frac{\alpha_{3}\left(\alpha_{4}-1\right)+\alpha_{5}\left(1-\alpha_{1}\right)}{\alpha_{3} \alpha_{6}-\alpha_{2} \alpha_{5}}\right) \\
& \quad\left|\frac{\left(\alpha_{3} \alpha_{6}-\alpha_{2} \alpha_{5}\right)\left(\alpha_{3}\left(\alpha_{6}\left(\alpha_{1}+\alpha_{4}\right)+\alpha_{2}\left(1-\alpha_{4}\right)\right)+\alpha_{5}\left(\alpha_{6}\left(1-\alpha_{1}\right)-2 \alpha_{2}\right)\right)}{\left(\alpha_{1} \alpha_{3} \alpha_{6}-\alpha_{2} \alpha_{5}+\alpha_{2} \alpha_{3}\left(\alpha_{4}-1\right)\right)\left(\alpha_{3} \alpha_{6} \alpha_{4}-\alpha_{2} \alpha_{5}+\alpha_{6} \alpha_{5}\left(1-\alpha_{1}\right)\right)}\right| \\
& \quad<1-\frac{\left(\alpha_{3} \alpha_{6}-\alpha_{2} \alpha_{5}\right)^{2}-\left(\alpha_{2} \alpha_{6}\left(\alpha_{1}-1\right)-\alpha_{2}^{2}\left(\alpha_{4}-1\right)\right)\left(\alpha_{3} \alpha_{5}\left(\alpha_{4}-1\right)-\alpha_{5}^{2}\left(\alpha_{1}-1\right)\right)}{\left(\alpha_{1} \alpha_{3} \alpha_{6}-\alpha_{2} \alpha_{5}+\alpha_{2} \alpha_{3}\left(\alpha_{4}-1\right)\right)\left(\alpha_{3} \alpha_{6} \alpha_{4}-\alpha_{2} \alpha_{5}+\alpha_{6} \alpha_{5}\left(1-\alpha_{1}\right)\right)}<2 .
\end{aligned}
$$

Similarly, one can prove (ii), (iii), and (iv).

\section{Bifurcations Analysis}

In this section, we will study the bifurcation analysis of discrete model (5) about the equilibria $O(0,0), A\left(\left(\alpha_{1}-\right.\right.$ $\left.1) / \alpha_{3}, 0\right), B\left(0,\left(\alpha_{4}-1\right) / \alpha_{6}\right)$ and $C\left(\left(\left(\alpha_{1}-1\right) \alpha_{6}-\alpha_{2}\left(\alpha_{4}-\right.\right.\right.$ $\left.1)) /\left(\alpha_{3} \alpha_{6}-\alpha_{2} \alpha_{5}\right),\left(\alpha_{3}\left(\alpha_{4}-1\right)+\alpha_{5}\left(1-\alpha_{1}\right)\right) /\left(\alpha_{3} \alpha_{6}-\alpha_{2} \alpha_{5}\right)\right)$. From theoretical results obtained in Section 3, we conclude the following:

(i) If condition (iv), that is, $\alpha_{1}=1$, of Theorem 3 holds, then one of the eigenvalues of $J_{O(0,0)}$ about $O(0,0)$ is 1 and so fold bifurcation may occur when parameters vary in a small neighborhood of $\alpha_{1}=1$. The condition (iv) of Theorem 3 can be rewritten as the following set:

$$
F_{\mathrm{O}(0,0)}=\left\{\left(\alpha_{1}, \alpha_{2}, \alpha_{3}, \alpha_{4}, \alpha_{5}, \alpha_{6}\right): \alpha_{1}=1\right\} .
$$

(ii) From Theorem 4, we can see that one of the eigenvalues of $J_{A\left(\left(\alpha_{1}-1\right) / \alpha_{3}, 0\right)}$ about $A\left(\left(\alpha_{1}-1\right) / \alpha_{3}, 0\right)$ is -1 and other is neither 1 nor -1 when the parameters of the discrete model (5) are located in the following set:

$$
\begin{aligned}
& F_{A\left(\left(\alpha_{1}-1\right) / \alpha_{3}, 0\right)} \\
& \quad=\left\{\left(\alpha_{1}, \alpha_{2}, \alpha_{3}, \alpha_{4}, \alpha_{5}, \alpha_{6}\right): \alpha_{5}=\frac{\alpha_{3}\left(\alpha_{4}+1\right)}{\alpha_{1}-1}\right\} .
\end{aligned}
$$

Therefore, $A\left(\left(\alpha_{1}-1\right) / \alpha_{3}, 0\right)$ can undergo flip or period-doubling bifurcation when all parameters of the discrete model (5) vary in a small neighborhood of $F_{A\left(\left(\alpha_{1}-1\right) / \alpha_{3}, 0\right)}$. When the parameters are in $F_{A\left(\left(\alpha_{1}-1\right) / \alpha_{3}, 0\right)}$, a center manifold of the discrete model (5) is $y=0$, and thus (5) restricted to this central manifold is

$$
x_{n+1}=\frac{\alpha_{1} x_{n}}{1+\alpha_{3} x_{n}} .
$$

This shows that the predator becomes extension and prey undergoes period-doubling bifurcation to chaos on choosing bifurcation parameter $\alpha_{5}$. 
(iii) From Theorem 5, it is easy to verify that one of the eigenvalues of $J_{B\left(0,\left(\alpha_{4}-1\right) / \alpha_{6}\right)}$ about $B\left(0,\left(\alpha_{4}-1\right) / \alpha_{6}\right)$ is -1 and the other is neither 1 nor -1 when the parameters of the discrete model (5) are located in the following set:

$$
\begin{aligned}
& F_{B\left(0,\left(\alpha_{4}-1\right) / \alpha_{6}\right)} \\
& \quad=\left\{\left(\alpha_{1}, \alpha_{2}, \alpha_{3}, \alpha_{4}, \alpha_{5}, \alpha_{6}\right): \alpha_{2}=\frac{\left(\alpha_{1}+1\right) \alpha_{6}}{\alpha_{4}-1}\right\} .
\end{aligned}
$$

Therefore, $B\left(0,\left(\alpha_{4}-1\right) / \alpha_{6}\right)$ undergoes perioddoubling bifurcation when all parameters of the discrete model (5) vary in a small neighborhood of $F_{B\left(0,\left(\alpha_{4}-1\right) / \alpha_{6}\right)}$. When the parameters are in $F_{B\left(0,\left(\alpha_{4}-1\right) / \alpha_{6}\right)}$, a center manifold of the discrete model (5) is $x=0$, and thus (5) restricted to this central manifold is

$$
y_{n+1}=\frac{\alpha_{4} y_{n}}{1+\alpha_{6} y_{n}}
$$

$$
\begin{aligned}
\Delta= & p^{2}-4 q, \\
= & \left(\frac{\left(\alpha_{3} \alpha_{6}-\alpha_{2} \alpha_{5}\right)\left(\alpha_{3}\left(\alpha_{6}\left(\alpha_{1}+\alpha_{4}\right)+\alpha_{2}\left(1-\alpha_{4}\right)\right)+\alpha_{5}\left(\alpha_{6}\left(1-\alpha_{1}\right)-2 \alpha_{2}\right)\right)}{\left(\alpha_{1} \alpha_{3} \alpha_{6}-\alpha_{2} \alpha_{5}+\alpha_{2} \alpha_{3}\left(\alpha_{4}-1\right)\right)\left(\alpha_{3} \alpha_{6} \alpha_{4}-\alpha_{2} \alpha_{5}+\alpha_{6} \alpha_{5}\left(1-\alpha_{1}\right)\right)}\right)^{2} \\
& -4\left(\frac{\left(\alpha_{3} \alpha_{6}-\alpha_{2} \alpha_{5}\right)^{2}-\left(\alpha_{2} \alpha_{6}\left(\alpha_{1}-1\right)-\alpha_{2}^{2}\left(\alpha_{4}-1\right)\right)\left(\alpha_{3} \alpha_{5}\left(\alpha_{4}-1\right)-\alpha_{5}^{2}\left(\alpha_{1}-1\right)\right)}{\left(\alpha_{1} \alpha_{3} \alpha_{6}-\alpha_{2} \alpha_{5}+\alpha_{2} \alpha_{3}\left(\alpha_{4}-1\right)\right)\left(\alpha_{3} \alpha_{6} \alpha_{4}-\alpha_{2} \alpha_{5}+\alpha_{6} \alpha_{5}\left(1-\alpha_{1}\right)\right)}\right) .
\end{aligned}
$$

Hereafter we state the topological classification about $C\left(\left(\left(\alpha_{1}-\right.\right.\right.$ 1) $\left.\alpha_{6}-\alpha_{2}\left(\alpha_{4}-1\right)\right) /\left(\alpha_{3} \alpha_{6}-\alpha_{2} \alpha_{5}\right),\left(\alpha_{3}\left(\alpha_{4}-1\right)+\alpha_{5}\left(1-\alpha_{1}\right)\right) /\left(\alpha_{3} \alpha_{6}-\right.$ $\left.\alpha_{2} \alpha_{5}\right)$ ) of system (5) as follows.
This shows that the prey becomes extension and predator undergoes period-doubling bifurcation to chaos on choosing bifurcation parameter $\alpha_{2}$.

Now before studying the bifurcation analysis of the discrete model (5) about the unique positive equilibrium point $C\left(\left(\left(\alpha_{1}-1\right) \alpha_{6}-\alpha_{2}\left(\alpha_{4}-1\right)\right) /\left(\alpha_{3} \alpha_{6}-\alpha_{2} \alpha_{5}\right)\right.$, $\left.\left(\alpha_{3}\left(\alpha_{4}-1\right)+\alpha_{5}\left(1-\alpha_{1}\right)\right) /\left(\alpha_{3} \alpha_{6}-\alpha_{2} \alpha_{5}\right)\right)$, first we will prove that there exist different topological types of this equilibrium point. Recall that eigenvalues of $J_{C\left(\left(\left(\alpha_{1}-1\right) \alpha_{6}-\alpha_{2}\left(\alpha_{4}-1\right)\right) /\left(\alpha_{3} \alpha_{6}-\alpha_{2} \alpha_{5}\right),\left(\alpha_{3}\left(\alpha_{4}-1\right)+\alpha_{5}\left(1-\alpha_{1}\right)\right) /\left(\alpha_{3} \alpha_{6}-\alpha_{2} \alpha_{5}\right)\right)}$ about

$$
\begin{gathered}
C\left(\frac{\left(\alpha_{1}-1\right) \alpha_{6}-\alpha_{2}\left(\alpha_{4}-1\right)}{\alpha_{3} \alpha_{6}-\alpha_{2} \alpha_{5}},\right. \\
\left.\frac{\alpha_{3}\left(\alpha_{4}-1\right)+\alpha_{5}\left(1-\alpha_{1}\right)}{\alpha_{3} \alpha_{6}-\alpha_{2} \alpha_{5}}\right)
\end{gathered}
$$

are

$$
\lambda_{1,2}=\frac{-p \pm \sqrt{\Delta}}{2}
$$

where

Theorem 8. For $C\left(\left(\left(\alpha_{1}-1\right) \alpha_{6}-\alpha_{2}\left(\alpha_{4}-1\right)\right) /\left(\alpha_{3} \alpha_{6}-\alpha_{2} \alpha_{5}\right)\right.$, $\left.\left(\alpha_{3}\left(\alpha_{4}-1\right)+\alpha_{5}\left(1-\alpha_{1}\right)\right) /\left(\alpha_{3} \alpha_{6}-\alpha_{2} \alpha_{5}\right)\right)$ of system (5), the following topological classification holds:

(i) $C\left(\left(\left(\alpha_{1}-1\right) \alpha_{6}-\alpha_{2}\left(\alpha_{4}-1\right)\right) /\left(\alpha_{3} \alpha_{6}-\alpha_{2} \alpha_{5}\right),\left(\alpha_{3}\left(\alpha_{4}-1\right)+\right.\right.$ $\left.\left.\alpha_{5}\left(1-\alpha_{1}\right)\right) /\left(\alpha_{3} \alpha_{6}-\alpha_{2} \alpha_{5}\right)\right)$ is a locally asymptotically stable focus if $\Delta<0$ and

$$
\frac{\left(\alpha_{3} \alpha_{6}-\alpha_{2} \alpha_{5}\right)^{2}-\left(\alpha_{2} \alpha_{6}\left(\alpha_{1}-1\right)-\alpha_{2}^{2}\left(\alpha_{4}-1\right)\right)\left(\alpha_{3} \alpha_{5}\left(\alpha_{4}-1\right)-\alpha_{5}^{2}\left(\alpha_{1}-1\right)\right)}{\left(\alpha_{1} \alpha_{3} \alpha_{6}-\alpha_{2} \alpha_{5}+\alpha_{2} \alpha_{3}\left(\alpha_{4}-1\right)\right)\left(\alpha_{3} \alpha_{6} \alpha_{4}-\alpha_{2} \alpha_{5}+\alpha_{6} \alpha_{5}\left(1-\alpha_{1}\right)\right)}<1
$$

(ii) $C\left(\left(\left(\alpha_{1}-1\right) \alpha_{6}-\alpha_{2}\left(\alpha_{4}-1\right)\right) /\left(\alpha_{3} \alpha_{6}-\alpha_{2} \alpha_{5}\right),\left(\alpha_{3}\left(\alpha_{4}-\right.\right.\right.$ 1) $\left.\left.+\alpha_{5}\left(1-\alpha_{1}\right)\right) /\left(\alpha_{3} \alpha_{6}-\alpha_{2} \alpha_{5}\right)\right)$ is an unstable focus if $\Delta<0$ and

$$
\frac{\left(\alpha_{3} \alpha_{6}-\alpha_{2} \alpha_{5}\right)^{2}-\left(\alpha_{2} \alpha_{6}\left(\alpha_{1}-1\right)-\alpha_{2}^{2}\left(\alpha_{4}-1\right)\right)\left(\alpha_{3} \alpha_{5}\left(\alpha_{4}-1\right)-\alpha_{5}^{2}\left(\alpha_{1}-1\right)\right)}{\left(\alpha_{1} \alpha_{3} \alpha_{6}-\alpha_{2} \alpha_{5}+\alpha_{2} \alpha_{3}\left(\alpha_{4}-1\right)\right)\left(\alpha_{3} \alpha_{6} \alpha_{4}-\alpha_{2} \alpha_{5}+\alpha_{6} \alpha_{5}\left(1-\alpha_{1}\right)\right)}>1
$$


(iii) $C\left(\left(\left(\alpha_{1}-1\right) \alpha_{6}-\alpha_{2}\left(\alpha_{4}-1\right)\right) /\left(\alpha_{3} \alpha_{6}-\alpha_{2} \alpha_{5}\right),\left(\alpha_{3}\left(\alpha_{4}-\right.\right.\right.$ 1) $\left.\left.+\alpha_{5}\left(1-\alpha_{1}\right)\right) /\left(\alpha_{3} \alpha_{6}-\alpha_{2} \alpha_{5}\right)\right)$ is nonhyperbolic if $\Delta<0$ and

$$
\frac{\left(\alpha_{3} \alpha_{6}-\alpha_{2} \alpha_{5}\right)^{2}-\left(\alpha_{2} \alpha_{6}\left(\alpha_{1}-1\right)-\alpha_{2}^{2}\left(\alpha_{4}-1\right)\right)\left(\alpha_{3} \alpha_{5}\left(\alpha_{4}-1\right)-\alpha_{5}^{2}\left(\alpha_{1}-1\right)\right)}{\left(\alpha_{1} \alpha_{3} \alpha_{6}-\alpha_{2} \alpha_{5}+\alpha_{2} \alpha_{3}\left(\alpha_{4}-1\right)\right)\left(\alpha_{3} \alpha_{6} \alpha_{4}-\alpha_{2} \alpha_{5}+\alpha_{6} \alpha_{5}\left(1-\alpha_{1}\right)\right)}=1 .
$$

We can see that the eigenvalues of are a pair of complex conjugates with modulus 1 when $J_{C\left(\left(\left(\alpha_{1}-1\right) \alpha_{6}-\alpha_{2}\left(\alpha_{4}-1\right)\right) /\left(\alpha_{3} \alpha_{6}-\alpha_{2} \alpha_{5}\right),\left(\alpha_{3}\left(\alpha_{4}-1\right)+\alpha_{5}\left(1-\alpha_{1}\right)\right) /\left(\alpha_{3} \alpha_{6}-\alpha_{2} \alpha_{5}\right)\right)}$ parameters of model (5) are located in the following set:

$$
\begin{aligned}
N_{C} & =\left\{\left(\alpha_{1}, \alpha_{2}, \alpha_{3}, \alpha_{4}, \alpha_{5}, \alpha_{6}\right): \Delta<0, \frac{\left(\alpha_{3} \alpha_{6}-\alpha_{2} \alpha_{5}\right)^{2}-\left(\alpha_{2} \alpha_{6}\left(\alpha_{1}-1\right)-\alpha_{2}^{2}\left(\alpha_{4}-1\right)\right)\left(\alpha_{3} \alpha_{5}\left(\alpha_{4}-1\right)-\alpha_{5}^{2}\left(\alpha_{1}-1\right)\right)}{\left(\alpha_{1} \alpha_{3} \alpha_{6}-\alpha_{2} \alpha_{5}+\alpha_{2} \alpha_{3}\left(\alpha_{4}-1\right)\right)\left(\alpha_{3} \alpha_{6} \alpha_{4}-\alpha_{2} \alpha_{5}+\alpha_{6} \alpha_{5}\left(1-\alpha_{1}\right)\right)}\right. \\
& =1\} .
\end{aligned}
$$

Therefore, $C\left(\left(\left(\alpha_{1}-1\right) \alpha_{6}-\alpha_{2}\left(\alpha_{4}-1\right)\right) /\left(\alpha_{3} \alpha_{6}-\alpha_{2} \alpha_{5}\right)\right.$, $\left.\left(\alpha_{3}\left(\alpha_{4}-1\right)+\alpha_{5}\left(1-\alpha_{1}\right)\right) /\left(\alpha_{3} \alpha_{6}-\alpha_{2} \alpha_{5}\right)\right)$ undergoes Neimark-Sacker bifurcation when all parameters of model (5) vary in a small neighborhood of

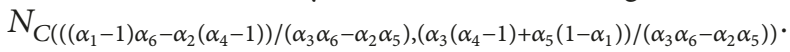

Hereafter we will study the Neimark-Sacker bifurcation of model (5) about

$$
\begin{gathered}
C\left(\frac{\left(\alpha_{1}-1\right) \alpha_{6}-\alpha_{2}\left(\alpha_{4}-1\right)}{\alpha_{3} \alpha_{6}-\alpha_{2} \alpha_{5}},\right. \\
\left.\frac{\alpha_{3}\left(\alpha_{4}-1\right)+\alpha_{5}\left(1-\alpha_{1}\right)}{\alpha_{3} \alpha_{6}-\alpha_{2} \alpha_{5}}\right) .
\end{gathered}
$$

For given parameters $\alpha_{1}, \alpha_{2}, \alpha_{3}, \alpha_{4}, \alpha_{5}, \alpha_{6}$, let

$$
\begin{gathered}
F\left(\alpha_{1}, \alpha_{2}, \alpha_{3}, \alpha_{4}, \alpha_{5}, \alpha_{6}\right)=\left(\alpha_{3} \alpha_{6}-\alpha_{2} \alpha_{5}\right)^{2} \\
-\left(\alpha_{2} \alpha_{6}\left(\alpha_{1}-1\right)-\alpha_{2}^{2}\left(\alpha_{4}-1\right)\right) \\
\cdot\left(\alpha_{3} \alpha_{5}\left(\alpha_{4}-1\right)-\alpha_{5}^{2}\left(\alpha_{1}-1\right)\right) \\
-\left(\alpha_{1} \alpha_{3} \alpha_{6}-\alpha_{2} \alpha_{5}+\alpha_{2} \alpha_{3}\left(\alpha_{4}-1\right)\right) \\
\cdot\left(\alpha_{3} \alpha_{6} \alpha_{4}-\alpha_{2} \alpha_{5}+\alpha_{6} \alpha_{5}\left(1-\alpha_{1}\right)\right) .
\end{gathered}
$$

$$
\begin{aligned}
& p(\epsilon)=\frac{\left(\alpha_{3} \alpha_{6}-\alpha_{2} \alpha_{5}\right)\left(\alpha_{3}\left(\alpha_{6}\left(\alpha_{1}^{*}+\epsilon+\alpha_{4}\right)+\alpha_{2}\left(1-\alpha_{4}\right)\right)+\alpha_{5}\left(\alpha_{6}\left(1-\alpha_{1}^{*}-\epsilon\right)-2 \alpha_{2}\right)\right)}{\left(\left(\alpha_{1}^{*}+\epsilon\right) \alpha_{3} \alpha_{6}-\alpha_{2} \alpha_{5}+\alpha_{2} \alpha_{3}\left(\alpha_{4}-1\right)\right)\left(\alpha_{3} \alpha_{6} \alpha_{4}-\alpha_{2} \alpha_{5}+\alpha_{6} \alpha_{5}\left(1-\alpha_{1}^{*}-\epsilon\right)\right)}, \\
& q(\epsilon)=\frac{\left(\alpha_{3} \alpha_{6}-\alpha_{2} \alpha_{5}\right)^{2}-\left(\alpha_{2} \alpha_{6}\left(\alpha_{1}^{*}+\epsilon-1\right)-\alpha_{2}^{2}\left(\alpha_{4}-1\right)\right)\left(\alpha_{3} \alpha_{5}\left(\alpha_{4}-1\right)-\alpha_{5}^{2}\left(\alpha_{1}^{*}+\epsilon-1\right)\right)}{\left(\left(\alpha_{1}^{*}+\epsilon\right) \alpha_{3} \alpha_{6}-\alpha_{2} \alpha_{5}+\alpha_{2} \alpha_{3}\left(\alpha_{4}-1\right)\right)\left(\alpha_{3} \alpha_{6} \alpha_{4}-\alpha_{2} \alpha_{5}+\alpha_{6} \alpha_{5}\left(1-\alpha_{1}^{*}-\epsilon\right)\right)} .
\end{aligned}
$$


The roots of characteristic equation of are

$J_{C\left(\left(\left(\alpha_{1}^{*}+\epsilon-1\right) \alpha_{6}-\alpha_{2}\left(\alpha_{4}-1\right)\right) /\left(\alpha_{3} \alpha_{6}-\alpha_{2} \alpha_{5}\right),\left(\alpha_{3}\left(\alpha_{4}-1\right)+\alpha_{5}\left(1-\alpha_{1}^{*}-\epsilon\right)\right) /\left(\alpha_{3} \alpha_{6}-\alpha_{2} \alpha_{5}\right)\right)}$

about

$$
\begin{gathered}
C\left(\frac{\left(\alpha_{1}^{*}+\epsilon-1\right) \alpha_{6}-\alpha_{2}\left(\alpha_{4}-1\right)}{\alpha_{3} \alpha_{6}-\alpha_{2} \alpha_{5}},\right. \\
\left.\frac{\alpha_{3}\left(\alpha_{4}-1\right)+\alpha_{5}\left(1-\alpha_{1}^{*}-\epsilon\right)}{\alpha_{3} \alpha_{6}-\alpha_{2} \alpha_{5}}\right)
\end{gathered}
$$

$$
\begin{aligned}
\lambda_{1,2} & =\frac{p(\epsilon) \pm \iota \sqrt{4 q(\epsilon)-p^{2}(\epsilon)}}{2}, \\
& =\frac{\left(\alpha_{3} \alpha_{6}-\alpha_{2} \alpha_{5}\right)\left(\alpha_{3}\left(\alpha_{6}\left(\alpha_{1}^{*}+\epsilon+\alpha_{4}\right)+\alpha_{2}\left(1-\alpha_{4}\right)\right)+\alpha_{5}\left(\alpha_{6}\left(1-\alpha_{1}^{*}-\epsilon\right)-2 \alpha_{2}\right)\right)}{2\left(\left(\alpha_{1}^{*}+\epsilon\right) \alpha_{3} \alpha_{6}-\alpha_{2} \alpha_{5}+\alpha_{2} \alpha_{3}\left(\alpha_{4}-1\right)\right)\left(\alpha_{3} \alpha_{6} \alpha_{4}-\alpha_{2} \alpha_{5}+\alpha_{6} \alpha_{5}\left(1-\alpha_{1}^{*}-\epsilon\right)\right)} \pm \frac{\iota}{2} \sqrt{\Gamma(\varepsilon)},
\end{aligned}
$$

where

$$
\begin{aligned}
\Gamma(\epsilon)= & 4 \frac{\left(\alpha_{3} \alpha_{6}-\alpha_{2} \alpha_{5}\right)^{2}-\left(\alpha_{2} \alpha_{6}\left(\alpha_{1}^{*}+\epsilon-1\right)-\alpha_{2}^{2}\left(\alpha_{4}-1\right)\right)\left(\alpha_{3} \alpha_{5}\left(\alpha_{4}-1\right)-\alpha_{5}^{2}\left(\alpha_{1}^{*}+\epsilon-1\right)\right)}{\left(\left(\alpha_{1}^{*}+\epsilon\right) \alpha_{3} \alpha_{6}-\alpha_{2} \alpha_{5}+\alpha_{2} \alpha_{3}\left(\alpha_{4}-1\right)\right)\left(\alpha_{3} \alpha_{6} \alpha_{4}-\alpha_{2} \alpha_{5}+\alpha_{6} \alpha_{5}\left(1-\alpha_{1}^{*}-\epsilon\right)\right)} \\
& -\left(\frac{\left(\alpha_{3} \alpha_{6}-\alpha_{2} \alpha_{5}\right)\left(\alpha_{3}\left(\alpha_{6}\left(\alpha_{1}^{*}+\epsilon+\alpha_{4}\right)+\alpha_{2}\left(1-\alpha_{4}\right)\right)+\alpha_{5}\left(\alpha_{6}\left(1-\alpha_{1}^{*}-\epsilon\right)-2 \alpha_{2}\right)\right)}{\left(\left(\alpha_{1}^{*}+\epsilon\right) \alpha_{3} \alpha_{6}-\alpha_{2} \alpha_{5}+\alpha_{2} \alpha_{3}\left(\alpha_{4}-1\right)\right)\left(\alpha_{3} \alpha_{6} \alpha_{4}-\alpha_{2} \alpha_{5}+\alpha_{6} \alpha_{5}\left(1-\alpha_{1}^{*}-\epsilon\right)\right)}\right)^{2} .
\end{aligned}
$$

Moreover $\left|\lambda_{1,2}\right|=\sqrt{q(\epsilon)}$ and $\left.\left(d\left|\lambda_{1,2}\right| / d \epsilon\right)\right|_{\epsilon=0} \neq 0$. Additionally, we required that when $\epsilon=0, \lambda_{1,2}^{m} \neq 1, m=1,2,3,4$, which corresponds to $p(0) \neq-2,0,1,2$. Since $p(0)^{2}-4 q(0)<$ 0 and $q(0)=1$, then $q(0)^{2}<4$ and hence $q(0) \neq \pm 2$. Thus, we only require that $q_{1}(0) \neq 0,1$, which holds true by computation.

Let $u_{n}=x_{n}-x^{*}, v_{n}=y_{n}-y^{*}$; then $C\left(\left(\left(\alpha_{1}-1\right) \alpha_{6}-\alpha_{2}\left(\alpha_{4}-\right.\right.\right.$ 1)) $\left./\left(\alpha_{3} \alpha_{6}-\alpha_{2} \alpha_{5}\right),\left(\alpha_{3}\left(\alpha_{4}-1\right)+\alpha_{5}\left(1-\alpha_{1}\right)\right) /\left(\alpha_{3} \alpha_{6}-\alpha_{2} \alpha_{5}\right)\right)$ of system (5) transforms into $O(0,0)$. By calculation, we obtain

$$
\begin{aligned}
u_{n+1}= & \frac{\left(\alpha_{1}^{*}+\epsilon\right)\left(u_{n}+x^{*}\right)-\alpha_{2}\left(u_{n}+x^{*}\right)\left(v_{n}+y^{*}\right)}{1+\alpha_{3}\left(u_{n}+x^{*}\right)} \\
& -x^{*} \\
v_{n+1}= & \frac{\alpha_{4}\left(v_{n}+y^{*}\right)-\alpha_{5}\left(u_{n}+x^{*}\right)\left(v_{n}+y^{*}\right)}{1+\alpha_{6}\left(v_{n}+y^{*}\right)}-y^{*},
\end{aligned}
$$

where $x^{*}=\left(\left(\alpha_{1}-1\right) \alpha_{6}-\alpha_{2}\left(\alpha_{4}-1\right)\right) /\left(\alpha_{3} \alpha_{6}-\alpha_{2} \alpha_{5}\right)$ and $y^{*}=$ $\left(\alpha_{3}\left(\alpha_{4}-1\right)+\alpha_{5}\left(1-\alpha_{1}\right)\right) /\left(\alpha_{3} \alpha_{6}-\alpha_{2} \alpha_{5}\right)$. Hereafter when $\epsilon=0$, normal form of system (48) is studied. Expanding (48) up to second-order about $\left(u_{n}, v_{n}\right)=(0,0)$ by Taylor series, we get

$$
\begin{aligned}
u_{n+1}= & m_{11} u_{n}+m_{12} v_{n}+m_{13} u_{n}^{2}+m_{14} u_{n} v_{n} \\
& +o\left(\left(\left|u_{n}\right|+\left|v_{n}\right|\right)^{2}\right)
\end{aligned}
$$

$$
\begin{aligned}
v_{n+1}= & m_{21} u_{n}+m_{22} v_{n}+m_{23} u_{n} v_{n}+m_{24} v_{n}^{2} \\
& +o\left(\left(\left|u_{n}\right|+\left|v_{n}\right|\right)^{2}\right),
\end{aligned}
$$

where

$$
\begin{aligned}
& m_{11}=\frac{\alpha_{1}^{*}-\alpha_{2} y^{*}}{\left(1+\alpha_{3} x^{*}\right)^{2}}, \\
& m_{12}=-\frac{\alpha_{2} x^{*}}{1+\alpha_{3} x^{*}}, \\
& m_{13}=-\frac{\alpha_{3}\left(\alpha_{1}^{*}-\alpha_{2} y^{*}\right)}{\left(1+\alpha_{3} x^{*}\right)^{3}}, \\
& m_{14}=-\frac{\alpha_{2}}{\left(1+\alpha_{3} x^{*}\right)^{2}}, \\
& m_{21}=-\frac{\alpha_{5} y^{*}}{1+\alpha_{6} y^{*}}, \\
& m_{22}=\frac{\alpha_{4}-\alpha_{5} x^{*}}{\left(1+\alpha_{6} y^{*}\right)^{2}}, \\
& m_{23}=-\frac{\alpha_{5}}{\left(1+\alpha_{6} y^{*}\right)^{2}}, \\
& m_{24}=-\frac{\alpha_{6}\left(\alpha_{4}-\alpha_{5} x^{*}\right)}{\left(1+\alpha_{6} y^{*}\right)^{3}} .
\end{aligned}
$$


Now, let

$$
\begin{aligned}
& \eta=\frac{\left(\alpha_{3} \alpha_{6}-\alpha_{2} \alpha_{5}\right)\left(\alpha_{3}\left(\alpha_{6}\left(\alpha_{1}^{*}+\alpha_{4}\right)+\alpha_{2}\left(1-\alpha_{4}\right)\right)+\alpha_{5}\left(\alpha_{6}\left(1-\alpha_{1}^{*}\right)-2 \alpha_{2}\right)\right)}{2\left(\alpha_{1}^{*} \alpha_{3} \alpha_{6}-\alpha_{2} \alpha_{5}+\alpha_{2} \alpha_{3}\left(\alpha_{4}-1\right)\right)\left(\alpha_{3} \alpha_{6} \alpha_{4}-\alpha_{2} \alpha_{5}+\alpha_{6} \alpha_{5}\left(1-\alpha_{1}^{*}\right)\right)}, \\
& \zeta=\frac{1}{2} \sqrt{\Gamma(0)},
\end{aligned}
$$

and invertible matrix $T$ is defined by

$$
T=\left(\begin{array}{cc}
m_{12} & 0 \\
\eta-m_{11} & -\zeta
\end{array}\right) .
$$

Using the following translation

$$
\left(\begin{array}{c}
u_{n} \\
v_{n}
\end{array}\right)=\left(\begin{array}{cc}
m_{12} & 0 \\
\eta-m_{11} & -\zeta
\end{array}\right)\left(\begin{array}{c}
X_{n} \\
Y_{n}
\end{array}\right)
$$

$$
\begin{aligned}
& F\left(X_{n}, Y_{n}\right)=l_{11} X_{n}^{2}+l_{12} X_{n} Y_{n}+o\left(\left(\left|X_{n}\right|+\left|Y_{n}\right|\right)^{2}\right), \\
& G\left(X_{n}, Y_{n}\right)=l_{21} X_{n}^{2}+l_{22} X_{n} Y_{n}+l_{23} Y_{n}^{2}+o\left(\left(\left|X_{n}\right|+\left|Y_{n}\right|\right)^{2}\right),
\end{aligned}
$$

(49) gives

$$
\left(\begin{array}{c}
X_{n+1} \\
Y_{n+1}
\end{array}\right)=\left(\begin{array}{cc}
\eta & -\zeta \\
\zeta & \eta
\end{array}\right)\left(\begin{array}{c}
X_{n} \\
Y_{n}
\end{array}\right)+\left(\begin{array}{c}
F\left(X_{n}, Y_{n}\right) \\
G\left(X_{n}, Y_{n}\right)
\end{array}\right)
$$

where

$$
\begin{array}{r}
l_{11}=m_{12} m_{13}+m_{14}\left(\eta-m_{11}\right), \\
l_{12}=-\zeta m_{14} \\
l_{21}=\frac{\left(\eta-m_{11}\right)}{\zeta}\left[m_{12}\left(m_{13}+\frac{m_{14}\left(\eta-m_{11}\right)}{m_{12}}-m_{23}\right)-m_{24}\left(\eta-m_{11}\right)\right], \\
l_{22}=-m_{12}\left(\frac{m_{14}\left(\eta-m_{11}\right)}{m_{12}}-m_{23}\right)+m_{24}\left(\eta-m_{11}\right), \\
l_{23}=-\zeta m_{24} .
\end{array}
$$

In addition,

$$
\begin{aligned}
& \left.F_{X_{n} X_{n}}\right|_{(0,0)}=2 l_{11}, \\
& \left.F_{X_{n} Y_{n}}\right|_{(0,0)}=l_{12} \text {, } \\
& \left.F_{Y_{n} Y_{n}}\right|_{(0,0)}=0 \text {, } \\
& \left.F_{X_{n} X_{n} X_{n}}\right|_{(0,0)}=\left.F_{X_{n} X_{n} Y_{n}}\right|_{(0,0)}=\left.F_{X_{n} Y_{n} Y_{n}}\right|_{(0,0)} \\
& =\left.F_{Y_{n} Y_{n} Y_{n}}\right|_{(0,0)}=0, \\
& \left.G_{X_{n} X_{n}}\right|_{(0,0)}=2 l_{21} \text {, } \\
& \left.G_{X_{n} Y_{n}}\right|_{(0,0)}=l_{22}, \\
& \left.G_{Y_{n} Y_{n}}\right|_{(0,0)}=2 l_{23} \text {, }
\end{aligned}
$$

$$
\begin{aligned}
\left.G_{X_{n} X_{n} X_{n}}\right|_{(0,0)} & =\left.G_{X_{n} X_{n} Y_{n}}\right|_{(0,0)}=\left.G_{X_{n} Y_{n} Y_{n}}\right|_{(0,0)} \\
& =\left.G_{Y_{n} Y_{n} Y_{n}}\right|_{(0,0)}=0 .
\end{aligned}
$$

To guarantee the supercritical Neimark-Sacker bifurcation for (54), we require the following discriminatory quantity, that is, $\Psi<0$ (see [19-23]):

$$
\begin{aligned}
\Psi= & -\operatorname{Re}\left[\frac{(1-2 \bar{\lambda}) \bar{\lambda}^{2}}{1-\lambda} \tau_{11} \tau_{20}\right]-\frac{1}{2}\left\|\tau_{11}\right\|^{2}-\left\|\tau_{02}\right\|^{2} \\
& +\operatorname{Re}\left(\bar{\lambda} \tau_{21}\right),
\end{aligned}
$$


where

$$
\begin{aligned}
\tau_{02} & =\frac{1}{8}\left[F_{X_{n} X_{n}}-F_{Y_{n} Y_{n}}+2 G_{X_{n} Y_{n}}\right. \\
& \left.+\iota\left(G_{X_{n} X_{n}}-G_{Y_{n} Y_{n}}+2 F_{X_{n} Y_{n}}\right)\right]\left.\right|_{(0,0)}, \\
\tau_{11} & =\left.\frac{1}{4}\left[F_{X_{n} X_{n}}+F_{Y_{n} Y_{n}}+\iota\left(G_{X_{n} X_{n}}+G_{Y_{n} Y_{n}}\right)\right]\right|_{(0,0)}, \\
\tau_{20} & =\frac{1}{8}\left[F_{X_{n} X_{n}}-F_{Y_{n} Y_{n}}+2 G_{X_{n} Y_{n}}\right. \\
& \left.+\iota\left(G_{X_{n} X_{n}}-G_{Y_{n} Y_{n}}-2 F_{X_{n} Y_{n}}\right)\right]\left.\right|_{(0,0)}, \\
\tau_{21} & =\frac{1}{16}\left[F_{X_{n} X_{n} X_{n}}+F_{X_{n} Y_{n} Y_{n}}+G_{X_{n} X_{n} Y_{n}}+G_{Y_{n} Y_{n} Y_{n}}\right. \\
& \left.+\iota\left(G_{X_{n} X_{n} X_{n}}+G_{X_{n} Y_{n} Y_{n}}-F_{X_{n} X_{n} Y_{n}}-F_{Y_{n} Y_{n} Y_{n}}\right)\right]\left.\right|_{(0,0)} .
\end{aligned}
$$

A calculation reveals

$$
\begin{aligned}
& \tau_{02}=\frac{1}{4}\left[l_{11}+l_{22}+\iota\left(l_{21}-l_{23}+l_{12}\right)\right], \\
& \tau_{11}=\frac{1}{2}\left[l_{11}+\iota\left(l_{21}+l_{23}\right)\right], \\
& \tau_{20}=\frac{1}{4}\left[l_{11}+l_{22}+\iota\left(l_{21}-l_{23}-l_{12}\right)\right], \\
& \tau_{21}=0 .
\end{aligned}
$$

Based on this analysis and Neimark-Sacker bifurcation Theorem discussed in $[19,20]$, we reach the following Theorem.

Theorem 9. If $\Psi \neq 0$, then discrete model (5) undergoes a Neimark-Sacker bifurcation about

$$
\begin{gathered}
C\left(\frac{\left(\alpha_{1}-1\right) \alpha_{6}-\alpha_{2}\left(\alpha_{4}-1\right)}{\alpha_{3} \alpha_{6}-\alpha_{2} \alpha_{5}},\right. \\
\left.\frac{\alpha_{3}\left(\alpha_{4}-1\right)+\alpha_{5}\left(1-\alpha_{1}\right)}{\alpha_{3} \alpha_{6}-\alpha_{2} \alpha_{5}}\right)
\end{gathered}
$$

as the parameters $\left(\alpha_{1}, \alpha_{2}, \alpha_{3}, \alpha_{4}, \alpha_{5}, \alpha_{6}\right)$ go through $N_{C\left(\left(\left(\alpha_{1}-1\right) \alpha_{6}-\alpha_{2}\left(\alpha_{4}-1\right)\right) /\left(\alpha_{3} \alpha_{6}-\alpha_{2} \alpha_{5}\right),\left(\alpha_{3}\left(\alpha_{4}-1\right)+\alpha_{5}\left(1-\alpha_{1}\right)\right) /\left(\alpha_{3} \alpha_{6}-\alpha_{2} \alpha_{5}\right)\right)}$. Additionally, attracting (resp., repelling) invariant closed curve bifurcates from the unique positive equilibrium $C\left(\left(\left(\alpha_{1}-1\right) \alpha_{6}-\right.\right.$ $\left.\left.\alpha_{2}\left(\alpha_{4}-1\right)\right) /\left(\alpha_{3} \alpha_{6}-\alpha_{2} \alpha_{5}\right),\left(\alpha_{3}\left(\alpha_{4}-1\right)+\alpha_{5}\left(1-\alpha_{1}\right)\right) /\left(\alpha_{3} \alpha_{6}-\alpha_{2} \alpha_{5}\right)\right)$ if $\Psi<0$ (resp., $\Psi>0$ ).

\section{Boundedness and Construction of Invariant Rectangle}

In this section we will study boundedness character and construction of invariant rectangle of positive solution of the discrete model (5).

Theorem 10. For every positive solution $\left\{\left(x_{n}, y_{n}\right)\right\}_{n=0}^{\infty}$ of the discrete model (5), the following holds:

(i) Every positive solution of the discrete model (5) is bounded.

(ii) The set $\left[0, \alpha_{1} / \alpha_{3}\right] \times\left[0, \alpha_{4} / \alpha_{6}\right]$ is an invariant rectangle.
Proof. (i) Let $\left\{\left(x_{n}, y_{n}\right)\right\}_{n=0}^{\infty}$ be any positive solution of the discrete model (5). From (5), we have

$$
\begin{aligned}
x_{n+1}= & \frac{\alpha_{1} x_{n}-\alpha_{2} x_{n} y_{n}}{1+\alpha_{3} x_{n}} \\
< & \frac{\alpha_{1} x_{n}}{1+\alpha_{3} x_{n}}<\frac{\alpha_{1}}{\alpha_{3}}, \\
y_{n+1}= & \frac{\alpha_{4} y_{n}-\alpha_{5} x_{n} y_{n}}{1+\alpha_{6} y_{n}} \\
& <\frac{\alpha_{4} y_{n}}{1+\alpha_{6} y_{n}}<\frac{\alpha_{4}}{\alpha_{6}} .
\end{aligned}
$$

Hence, for every solution $\left\{\left(x_{n}, y_{n}\right)\right\}$ of the discrete model (5), one has

$$
\begin{aligned}
& 0 \leq x_{n}<\frac{\alpha_{1}}{\alpha_{3}}, \\
& 0 \leq y_{n}<\frac{\alpha_{4}}{\alpha_{6}} .
\end{aligned}
$$

(ii) For any positive solution $\left\{\left(x_{n}, y_{n}\right)\right\}_{n=0}^{\infty}$ of the discrete model (5) with initial conditions $x_{0} \in\left[0, \alpha_{1} / \alpha_{3}\right]$ and $y_{0} \in$ $\left[0, \alpha_{4} / \alpha_{6}\right]$, we have from (5)

$$
\begin{aligned}
0 & \leq x_{1}=\frac{\alpha_{1} x_{0}-\alpha_{2} x_{0} y_{0}}{1+\alpha_{3} x_{0}} \\
& <\frac{\alpha_{1} x_{0}}{1+\alpha_{3} x_{0}}<\frac{\alpha_{1}}{\alpha_{3}}, \\
0 & \leq y_{1}=\frac{\alpha_{4} y_{0}-\alpha_{5} x_{0} y_{0}}{1+\alpha_{6} y_{0}} \\
& <\frac{\alpha_{4} y_{0}}{1+\alpha_{6} y_{0}}<\frac{\alpha_{4}}{\alpha_{6}} .
\end{aligned}
$$

Hence, $x_{1} \in\left[0, \alpha_{1} / \alpha_{3}\right]$ and $y_{1} \in\left[0, \alpha_{4} / \alpha_{6}\right]$. Similarly, one can show that if $x_{k} \in\left[0, \alpha_{1} / \alpha_{3}\right]$ and $y_{k} \in\left[0, \alpha_{4} / \alpha_{6}\right]$, then $x_{k+1} \in\left[0, \alpha_{1} / \alpha_{3}\right]$ and $y_{k+1} \in\left[0, \alpha_{4} / \alpha_{6}\right]$.

\section{Global Stability}

Now in the following we will investigate global dynamics of the discrete model $(5)$ about $O(0,0)$ and the unique positive equilibrium $C\left(\left(\left(\alpha_{1}-1\right) \alpha_{6}-\alpha_{2}\left(\alpha_{4}-1\right)\right) /\left(\alpha_{3} \alpha_{6}-\alpha_{2} \alpha_{5}\right),\left(\alpha_{3}\left(\alpha_{4}-\right.\right.\right.$ 1) $\left.\left.+\alpha_{5}\left(1-\alpha_{1}\right)\right) /\left(\alpha_{3} \alpha_{6}-\alpha_{2} \alpha_{5}\right)\right)$.

Theorem 11. If $\alpha_{1}<1$ and $\alpha_{4}<1$, then equilibrium $O(0,0)$ of the discrete model (5) is globally asymptotically stable.

Proof. According to the conclusion (i) in Lemma 1, discrete model (5) has a unique equilibrium $O(0,0)$ in the first quadrant $\mathbb{R}^{2}$ and is a sink by Theorem 3 . Moreover every 
positive solution of the discrete model (5) in the first quadrant $\mathbb{R}^{2}$ satisfies

$$
\begin{aligned}
0 & <x_{n+1} \leq \frac{\alpha_{1} x_{n}}{1+\alpha_{3} x_{n}}=x_{n}\left(1-\frac{\alpha_{3} x_{n}+1-\alpha_{1}}{1+\alpha_{3} x_{n}}\right) \\
& <x_{n}, \\
0 & <y_{n+1} \leq \frac{\alpha_{4} y_{n}}{1+\alpha_{6} y_{n}}=y_{n}\left(1-\frac{\alpha_{6} y_{n}+1-\alpha_{4}}{1+\alpha_{6} y_{n}}\right) \\
& <y_{n},
\end{aligned}
$$

which leads to $\lim _{n \rightarrow \infty} x_{n}=0$ and $\lim _{n \rightarrow \infty} y_{n}=0$. Hence the boundary equilibrium $\mathrm{O}(0,0)$ of the discrete model (5) is globally asymptotically stable in the first quadrant $\mathbb{R}^{2}$.

Hereafter we use Theorem 1.16 of [14] to determine the global dynamics of the discrete model (5) about $C\left(\left(\left(\alpha_{1}-\right.\right.\right.$ 1) $\left.\alpha_{6}-\alpha_{2}\left(\alpha_{4}-1\right)\right) /\left(\alpha_{3} \alpha_{6}-\alpha_{2} \alpha_{5}\right),\left(\alpha_{3}\left(\alpha_{4}-1\right)+\alpha_{5}\left(1-\alpha_{1}\right)\right) /\left(\alpha_{3} \alpha_{6}-\right.$ $\left.\alpha_{2} \alpha_{5}\right)$ ).

Theorem 12. The unique positive equilibrium point $C\left(\left(\left(\alpha_{1}-\right.\right.\right.$ 1) $\left.\alpha_{6}-\alpha_{2}\left(\alpha_{4}-1\right)\right) /\left(\alpha_{3} \alpha_{6}-\alpha_{2} \alpha_{5}\right),\left(\alpha_{3}\left(\alpha_{4}-1\right)+\alpha_{5}\left(1-\alpha_{1}\right)\right) /\left(\alpha_{3} \alpha_{6}-\right.$ $\left.\alpha_{2} \alpha_{5}\right)$ ) of the discrete model (5) is a global attractor.

Proof. Let $f(x, y)=\left(\alpha_{1} x-\alpha_{2} x y\right) /\left(1+\alpha_{3} x\right)$ and $g(x, y)=$ $\left(\alpha_{4} y-\alpha_{5} x y\right) /\left(1+\alpha_{6} \bar{y}\right)$. Then, it is easy to see that $f(x, y)$ is nondecreasing in $x$ and nonincreasing in $y$ for every $(x, y) \in$ $\left[0, \alpha_{1} / \alpha_{3}\right] \times\left[0, \alpha_{4} / \alpha_{6}\right]$. Moreover, $g(x, y)$ is nonincreasing in $x$ and nondecreasing in $y$ for each $(x, y) \in\left[0, \alpha_{1} / \alpha_{3}\right] \times$ $\left[0, \alpha_{4} / \alpha_{6}\right]$. Let $\left(m_{1}, M_{1}, m_{2}, M_{2}\right)$ be a positive solution of system

$$
\begin{aligned}
& m_{1}=f\left(m_{1}, M_{2}\right), \\
& M_{1}=f\left(M_{1}, m_{2}\right), \\
& m_{2}=g\left(M_{1}, m_{2}\right), \\
& M_{2}=g\left(m_{1}, M_{2}\right) .
\end{aligned}
$$

Then, one has

$$
\begin{aligned}
& m_{1}=\frac{\alpha_{1} m_{1}-\alpha_{2} m_{1} M_{2}}{1+\alpha_{3} m_{1}}, \\
& M_{1}=\frac{\alpha_{1} M_{1}-\alpha_{2} M_{1} m_{2}}{1+\alpha_{3} M_{1}}, \\
& m_{2}=\frac{\alpha_{4} m_{2}-\alpha_{5} M_{1} m_{2}}{1+\alpha_{6} m_{2}}, \\
& M_{2}=\frac{\alpha_{4} M_{2}-\alpha_{5} m_{1} M_{2}}{1+\alpha_{6} M_{2}} .
\end{aligned}
$$

From (66), one has

$$
\begin{aligned}
& 1+\alpha_{3} m_{1}=\alpha_{1}-\alpha_{2} M_{2} \\
& 1+\alpha_{3} M_{1}=\alpha_{1}-\alpha_{2} m_{2} .
\end{aligned}
$$

From (67), one has

$$
\begin{aligned}
& 1+\alpha_{6} m_{2}=\alpha_{4}-\alpha_{5} M_{1}, \\
& 1+\alpha_{6} M_{2}=\alpha_{4}-\alpha_{5} m_{1} .
\end{aligned}
$$

On subtracting (68), one gets

$$
\alpha_{3}\left(m_{1}-M_{1}\right)=\alpha_{2}\left(m_{2}-M_{2}\right) \text {. }
$$

Similarly, subtracting (69) one gets

$$
\alpha_{6}\left(m_{2}-M_{2}\right)=\alpha_{5}\left(m_{1}-M_{1}\right) .
$$

From (70) and (71) one gets $\left(\alpha_{6} \alpha_{3}-\alpha_{2} \alpha_{4}\right)\left(m_{1}-M_{1}\right)=0$. Hence, $m_{1}=M_{1}$, and similarly one has $m_{2}=M_{2}$. Hence, from Theorem 1.16 of [14] the equilibrium $C\left(\left(\left(\alpha_{1}-1\right) \alpha_{6}-\right.\right.$ $\left.\left.\alpha_{2}\left(\alpha_{4}-1\right)\right) /\left(\alpha_{3} \alpha_{6}-\alpha_{2} \alpha_{5}\right),\left(\alpha_{3}\left(\alpha_{4}-1\right)+\alpha_{5}\left(1-\alpha_{1}\right)\right) /\left(\alpha_{3} \alpha_{6}-\alpha_{2} \alpha_{5}\right)\right)$ of the discrete model (5) is a global attractor.

Corollary 13. Under the conditions (10) and (18), the unique positive equilibrium point

$$
\begin{gathered}
C\left(\frac{\left(\alpha_{1}-1\right) \alpha_{6}-\alpha_{2}\left(\alpha_{4}-1\right)}{\alpha_{3} \alpha_{6}-\alpha_{2} \alpha_{5}},\right. \\
\left.\frac{\alpha_{3}\left(\alpha_{4}-1\right)+\alpha_{5}\left(1-\alpha_{1}\right)}{\alpha_{3} \alpha_{6}-\alpha_{2} \alpha_{5}}\right)
\end{gathered}
$$

of the discrete model (5) is globally asymptotically stable.

\section{Numerical Simulations}

In the following we present numerical simulations that represent different types of qualitative behavior of the discrete model (5).

Example 1. If $\alpha_{1}=2, \alpha_{2}=0.0007, \alpha_{3}=0.23, \alpha_{4}=7, \alpha_{5}=$ $0.3, \alpha_{6}=0.4$, then discrete model (5) with initial values $x_{0}=$ $0.007, y_{0}=0.0009$ can be written as

$$
\begin{aligned}
& x_{n+1}=\frac{2 x_{n}-0.0007 x_{n} y_{n}}{1+0.23 x_{n}} \\
& y_{n+1}=\frac{7 y_{n}-0.3 x_{n} y_{n}}{1+0.4 y_{n}} .
\end{aligned}
$$

In this case $\alpha_{1}=2>1, \alpha_{4}=7>1, \alpha_{3} \alpha_{6}=0.092>$ $\alpha_{2} \alpha_{5}=0.00021, \alpha_{6}=0.4>\alpha_{2}\left(\alpha_{4}-1\right) /\left(\alpha_{1}-1\right)=0.0042$, $\alpha_{5}=0.3<\alpha_{3}\left(\alpha_{4}-1\right) /\left(\alpha_{11}-1\right)=1.38$. This shows the correctness of the conditions for the unique positive equilibrium. A straightforward computation shows that condition (10) of Theorem 6, that is, $\Theta=0.07435317508000001$ $<\left(\alpha_{2} \alpha_{3}-\alpha_{2} \alpha_{3} \alpha_{4}-\alpha_{2} \alpha_{5}+\alpha_{1} \alpha_{3} \alpha_{6}\right)\left(-\alpha_{2} \alpha_{5}+\alpha_{3} \alpha_{4} \alpha_{6}+\right.$ $\left.\alpha_{5} \alpha_{6}-\alpha_{1} \alpha_{5} \alpha_{6}\right)=0.0957613829600004$, under which the unique positive equilibrium point is locally asymptotically stable, holds. Moreover for these arbitrary chosen values of parameters, the necessary and sufficient condition, under which the unique positive equilibrium point is locally asymptotically stable, is also satisfied; that is, $\mid\left(\alpha_{3} \alpha_{6}-\right.$ $\left.\alpha_{2} \alpha_{5}\right)\left(\alpha_{3}\left(\alpha_{6}\left(\alpha_{1}+\alpha_{4}\right)+\alpha_{2}\left(1-\alpha_{4}\right)\right)+\alpha_{5}\left(\alpha_{6}\left(1-\alpha_{1}\right)-2 \alpha_{2}\right)\right) /$ 


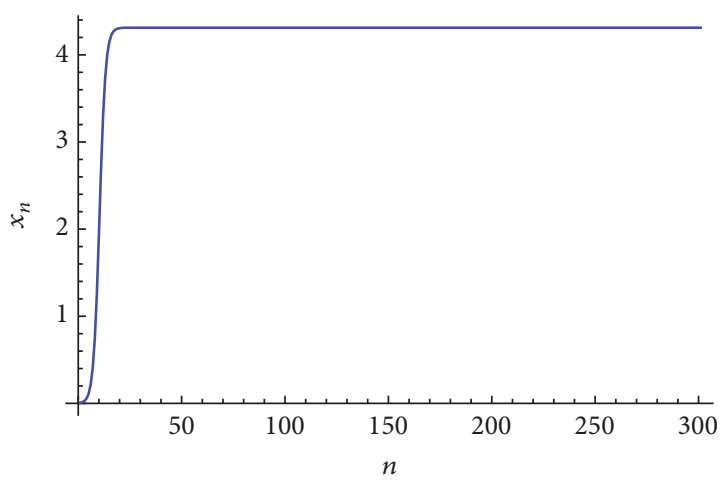

(a) Plot of $x_{n}$ for system (73)

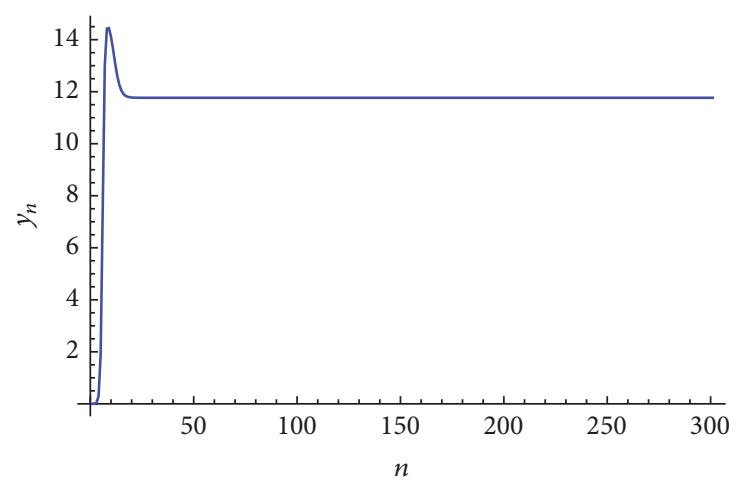

(b) Plot of $y_{n}$ for system (73)

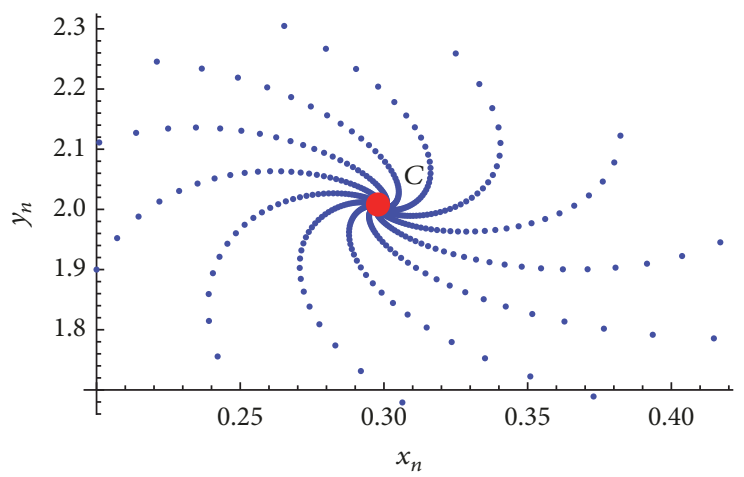

(c) Attractor of system (73)

Figure 1: Plots for system (73).

$\left(\alpha_{1} \alpha_{3} \alpha_{6}-\alpha_{2} \alpha_{5}+\alpha_{2} \alpha_{3}\left(\alpha_{4}-1\right)\right)\left(\alpha_{3} \alpha_{6} \alpha_{4}-\alpha_{2} \alpha_{5}+\alpha_{6} \alpha_{5}\left(1-\alpha_{1}\right)\right) \mid=$ $0.6702268987306942<1-\left(\left(\alpha_{3} \alpha_{6}-\alpha_{2} \alpha_{5}\right)^{2}-\left(\alpha_{2} \alpha_{6}\left(\alpha_{1}-1\right)-\right.\right.$ $\left.\left.\alpha_{2}^{2}\left(\alpha_{4}-1\right)\right)\left(\alpha_{3} \alpha_{5}\left(\alpha_{4}-1\right)-\alpha_{5}^{2}\left(\alpha_{1}-1\right)\right)\right) /\left(\alpha_{1} \alpha_{3} \alpha_{6}-\alpha_{2} \alpha_{5}+\alpha_{2} \alpha_{3}\left(\alpha_{4}-\right.\right.$ 1)) $\left(\alpha_{3} \alpha_{6} \alpha_{4}-\alpha_{2} \alpha_{5}+\alpha_{6} \alpha_{5}\left(1-\alpha_{1}\right)\right)=0.9138643327940412<$ 2. This verifies the condition for which the unique positive equilibrium is locally asymptotically stable. Also $0 \leq x_{n}<$ $\alpha_{1} / \alpha_{3}=8.695652173913043,0 \leq y_{n}<\alpha_{4} / \alpha_{6}=17.5$, and hence the parametric conditions under which every positive solution is bounded hold true. Moreover, in Figure 1 the plot of $x_{n}$ is shown in Figure 1(a), the plot of $y_{n}$ is shown in Figure 1(b), and attractor of system (73) is shown in Figure 1(c).

Example 2. If $\alpha_{1}=8, \alpha_{2}=0.00007, \alpha_{3}=3.23, \alpha_{4}=$ 7, $\alpha_{5}=1.3, \alpha_{6}=3.4$, then discrete model (5) with initial values $x_{0}=0.007, y_{0}=0.08$ can be written as

$$
\begin{aligned}
& x_{n+1}=\frac{8 x_{n}-0.00007 x_{n} y_{n}}{1+3.23 x_{n}} \\
& y_{n+1}=\frac{7 y_{n}-1.3 x_{n} y_{n}}{1+3.4 y_{n}} .
\end{aligned}
$$

In this case $\alpha_{1}=8>1, \alpha_{4}=7>1, \alpha_{3} \alpha_{6}=10.982>$ $\alpha_{2} \alpha_{5}=0.000091, \alpha_{6}=3.4>\alpha_{2}\left(\alpha_{4}-1\right) /\left(\alpha_{1}-1\right)=$ $0.00006, \alpha_{5}=1.3<\alpha_{3}\left(\alpha_{4}-1\right) /\left(\alpha_{11}-1\right)=2.76857$. This shows the correctness of the conditions of the unique positive equilibrium point. A straightforward computation shows that condition (10) of Theorem 6, that is, $\Theta=$ $4035.5030151773317<\left(\alpha_{2} \alpha_{3}-\alpha_{2} \alpha_{3} \alpha_{4}-\alpha_{2} \alpha_{5}+\alpha_{1} \alpha_{3} \alpha_{6}\right)\left(-\alpha_{2} \alpha_{5}+\right.$ $\left.\alpha_{3} \alpha_{4} \alpha_{6}+\alpha_{5} \alpha_{6}-\alpha_{1} \alpha_{5} \alpha_{6}\right)=4035.5030151773317$, holds. Moreover the necessary and sufficient condition under which the unique positive equilibrium point of the system is locally asymptotically stable is also satisfied; that is, $\mid\left(\alpha_{3} \alpha_{6}-\right.$ $\left.\alpha_{2} \alpha_{5}\right)\left(\alpha_{3}\left(\alpha_{6}\left(\alpha_{1}+\alpha_{4}\right)+\alpha_{2}\left(1-\alpha_{4}\right)\right)+\alpha_{5}\left(\alpha_{6}\left(1-\alpha_{1}\right)-2 \alpha_{2}\right)\right) /$ $\left(\alpha_{1} \alpha_{3} \alpha_{6}-\alpha_{2} \alpha_{5}+\alpha_{2} \alpha_{3}\left(\alpha_{4}-1\right)\right)\left(\alpha_{3} \alpha_{6} \alpha_{4}-\alpha_{2} \alpha_{5}+\alpha_{6} \alpha_{5}\left(1-\alpha_{1}\right)\right)$ $=0.36407043421653185<1-\left(\left(\alpha_{3} \alpha_{6}-\alpha_{2} \alpha_{5}\right)^{2}-\left(\alpha_{2} \alpha_{6}\left(\alpha_{1}-1\right)-\right.\right.$ $\left.\left.\alpha_{2}^{2}\left(\alpha_{4}-1\right)\right)\left(\alpha_{3} \alpha_{5}\left(\alpha_{4}-1\right)-\alpha_{5}^{2}\left(\alpha_{1}-1\right)\right)\right) /\left(\alpha_{1} \alpha_{3} \alpha_{6}-\alpha_{2} \alpha_{5}+\alpha_{2} \alpha_{3}\left(\alpha_{4}-\right.\right.$ 1)) $\left(\alpha_{3} \alpha_{6} \alpha_{4}-\alpha_{2} \alpha_{5}+\alpha_{6} \alpha_{5}\left(1-\alpha_{1}\right)\right)=0.9701211132646083<$ 2. This verifies the condition for which the unique positive equilibrium is locally asymptotically stable. Also $0 \leq x_{n}<$ $\alpha_{1} / \alpha_{3}=2.476780185758514,0 \leq y_{n}<\alpha_{4} / \alpha_{6}=$ 2.058823529411765 , and hence the parametric conditions under which every positive solution is bounded hold true. Moreover, in Figure 2 the plot of $x_{n}$ is shown in Figure 2(a), the plot of $y_{n}$ is shown in Figure 2(b), and attractor of system (74) is shown in Figure 2(c).

Example 3. If $\alpha_{1}=15, \alpha_{2}=0.057, \alpha_{3}=6.23, \alpha_{4}=7, \alpha_{5}=$ $1.3, \alpha_{6}=3.4$, then discrete model (5) with initial values $x_{0}=$ $0.008, y_{0}=0.009$ can be written as

$$
\begin{aligned}
& x_{n+1}=\frac{15 x_{n}-0.057 x_{n} y_{n}}{1+6.23 x_{n}} \\
& y_{n+1}=\frac{7 y_{n}-1.3 x_{n} y_{n}}{1+3.4 y_{n}} .
\end{aligned}
$$




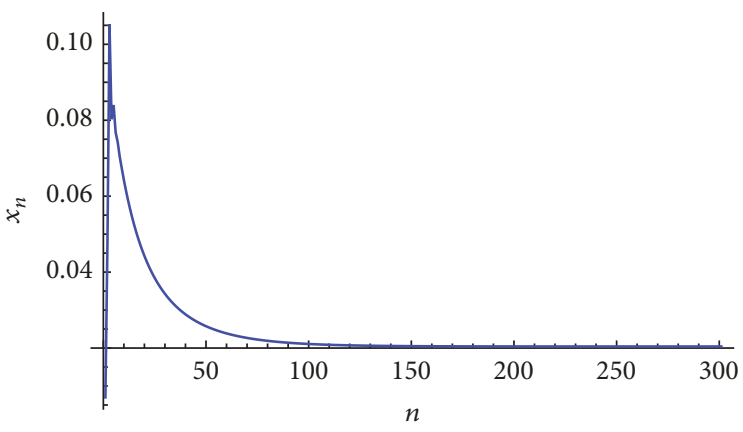

(a) Plot of $x_{n}$ for system (74)

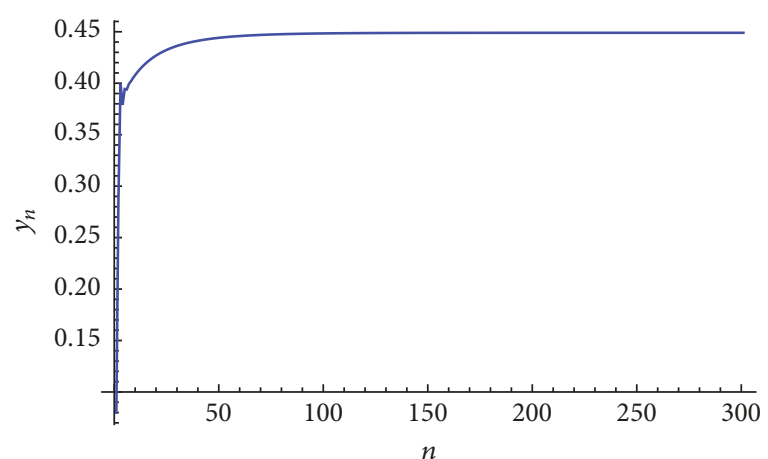

(b) Plot of $y_{n}$ for system (74)

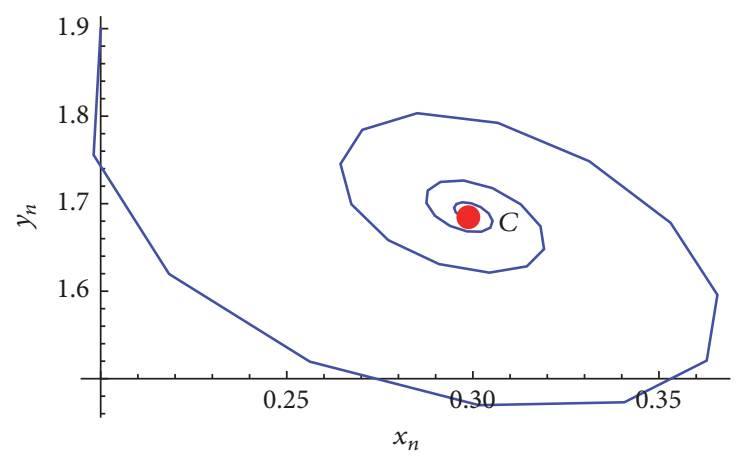

(c) Attractor of system (74)

Figure 2: Plots for system (74).

In this case $\alpha_{1}=15>1, \alpha_{4}=7>1, \alpha_{3} \alpha_{6}=$ $21.182>\alpha_{2} \alpha_{5}=0.0741, \alpha_{6}=3.4>\alpha_{2}\left(\alpha_{4}-1\right) /\left(\alpha_{1}-\right.$ $1=0.0244286, \alpha_{5}=1.3<\alpha_{3}\left(\alpha_{4}-1\right) /\left(\alpha_{1}-1\right)=$ 2.67. This shows the correctness of the conditions of the unique positive equilibrium point. A computation shows that condition (10) of Theorem 6, that is, $\Theta=9280.610848888002$ $<\left(\alpha_{2} \alpha_{3}-\alpha_{2} \alpha_{3} \alpha_{4}-\alpha_{2} \alpha_{5}+\alpha_{1} \alpha_{3} \alpha_{6}\right)\left(-\alpha_{2} \alpha_{5}+\alpha_{3} \alpha_{4} \alpha_{6}+\alpha_{5} \alpha_{6}-\right.$ $\left.\alpha_{1} \alpha_{5} \alpha_{6}\right)=27236.10716427601$, holds. Moreover for these arbitrary chosen values of parameters the necessary and sufficient condition, under which the unique positive equilibrium point is locally asymptotically stable, is also satisfied; that is, $\mid\left(\alpha_{3} \alpha_{6}-\alpha_{2} \alpha_{5}\right)\left(\alpha_{3}\left(\alpha_{6}\left(\alpha_{1}+\alpha_{4}\right)+\alpha_{2}\left(1-\alpha_{4}\right)\right)+\alpha_{5}\left(\alpha_{6}\left(1-\alpha_{1}\right)-2 \alpha_{2}\right)\right) /$ $\left(\alpha_{1} \alpha_{3} \alpha_{6}-\alpha_{2} \alpha_{5}+\alpha_{2} \alpha_{3}\left(\alpha_{4}-1\right)\right)\left(\alpha_{3} \alpha_{6} \alpha_{4}-\alpha_{2} \alpha_{5}+\alpha_{6} \alpha_{5}\left(1-\alpha_{1}\right)\right) \mid$ $=0.30727881013351915<1-\left(\left(\alpha_{3} \alpha_{6}-\alpha_{2} \alpha_{5}\right)^{2}-\left(\alpha_{2} \alpha_{6}\left(\alpha_{1}-1\right)-\right.\right.$ $\left.\left.\alpha_{2}^{2}\left(\alpha_{4}-1\right)\right)\left(\alpha_{3} \alpha_{5}\left(\alpha_{4}-1\right)-\alpha_{5}^{2}\left(\alpha_{1}-1\right)\right)\right) /\left(\alpha_{1} \alpha_{3} \alpha_{6}-\alpha_{2} \alpha_{5}+\alpha_{2} \alpha_{3}\left(\alpha_{4}-\right.\right.$ 1)) $\left(\alpha_{3} \alpha_{6} \alpha_{4}-\alpha_{2} \alpha_{5}+\alpha_{6} \alpha_{5}\left(1-\alpha_{1}\right)\right)=0.9862925899775977<$ 2. This verifies the condition for which the unique positive equilibrium is locally asymptotically stable. Also $0 \leq x_{n}<$ $\alpha_{1} / \alpha_{3}=2.407704654895666,0 \leq y_{n}<\alpha_{4} / \alpha_{6}=$ 2.058823529411765 , and hence the parametric conditions under which every positive solution is bounded hold true. Moreover, in Figure 3 the plot of $x_{n}$ is shown in Figure 3(a), the plot of $y_{n}$ is shown in Figure 3(b), and attractor of system (75) is shown in Figure 3(c).

\section{Conclusion}

This work is related to the global dynamics and bifurcations analysis of a two-dimensional discrete-time LotkaVolterra model in the closed first quadrant $\mathbb{R}^{2}$. We proved that the discrete model (5) has three boundary equilibria $O(0,0), A\left(\left(\alpha_{1}-1\right) / \alpha_{3}, 0\right), B\left(0,\left(\alpha_{4}-1\right) / \alpha_{6}\right)$ and the unique positive equilibrium

$$
\begin{gathered}
C\left(\frac{\left(\alpha_{1}-1\right) \alpha_{6}-\alpha_{2}\left(\alpha_{4}-1\right)}{\alpha_{3} \alpha_{6}-\alpha_{2} \alpha_{5}},\right. \\
\left.\frac{\alpha_{3}\left(\alpha_{4}-1\right)+\alpha_{5}\left(1-\alpha_{1}\right)}{\alpha_{3} \alpha_{6}-\alpha_{2} \alpha_{5}}\right)
\end{gathered}
$$

under certain parametric conditions. The method of linearization is used to prove the local asymptotic stability of these equilibria, and conclusions are presented in Table 1. We proved that boundary equilibrium $O(0,0)$ undergoes fold bifurcation when parameters vary in a small neighborhood of $\alpha_{1}=1$ and both $A\left(\left(\alpha_{1}-1\right) / \alpha_{3}, 0\right)$ and $B\left(0,\left(\alpha_{4}-1\right) / \alpha_{6}\right)$ undergo period-doubling bifurcation when parameters of the discrete model (5) are, respectively, located in the following sets:

$$
\begin{aligned}
& F_{A\left(\left(\alpha_{1}-1\right) / \alpha_{3}, 0\right)} \\
& \quad=\left\{\left(\alpha_{1}, \alpha_{2}, \alpha_{3}, \alpha_{4}, \alpha_{5}, \alpha_{6}\right): \alpha_{5}=\frac{\alpha_{3}\left(\alpha_{4}+1\right)}{\alpha_{1}-1}\right\}, \\
& F_{B\left(0,\left(\alpha_{4}-1\right) / \alpha_{6}\right)} \\
& \quad=\left\{\left(\alpha_{1}, \alpha_{2}, \alpha_{3}, \alpha_{4}, \alpha_{5}, \alpha_{6}\right): \alpha_{2}=\frac{\left(\alpha_{1}+1\right) \alpha_{6}}{\alpha_{4}-1}\right\} .
\end{aligned}
$$

We have also shown that $C\left(\left(\left(\alpha_{1}-1\right) \alpha_{6}-\alpha_{2}\left(\alpha_{4}-1\right)\right) /\left(\alpha_{3} \alpha_{6}-\right.\right.$ $\left.\left.\alpha_{2} \alpha_{5}\right),\left(\alpha_{3}\left(\alpha_{4}-1\right)+\alpha_{5}\left(1-\alpha_{1}\right)\right) /\left(\alpha_{3} \alpha_{6}-\alpha_{2} \alpha_{5}\right)\right)$ undergoes 


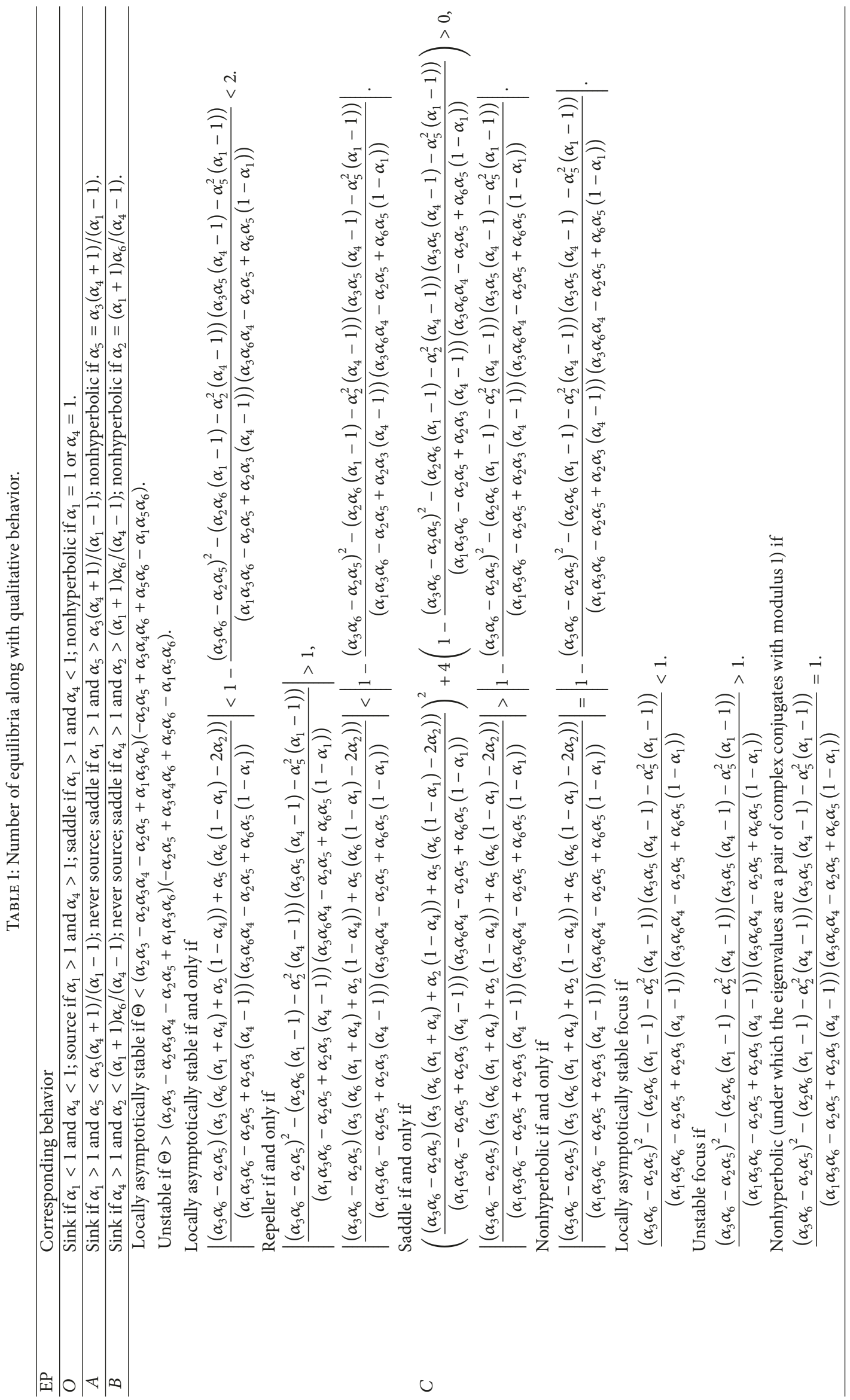




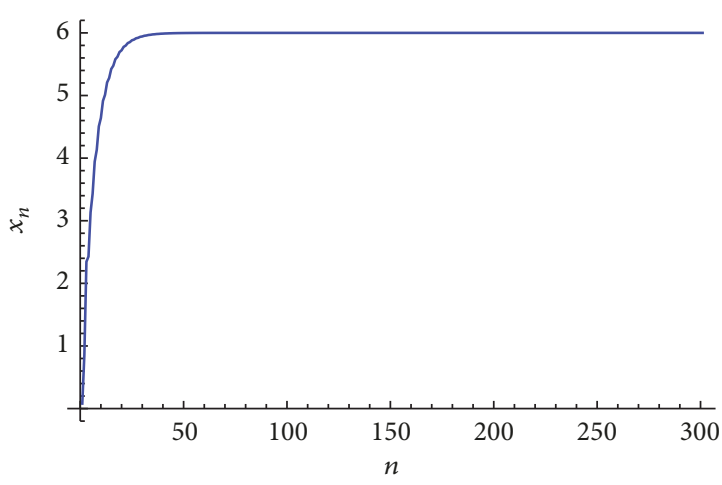

(a) Plot of $x_{n}$ for system (75)

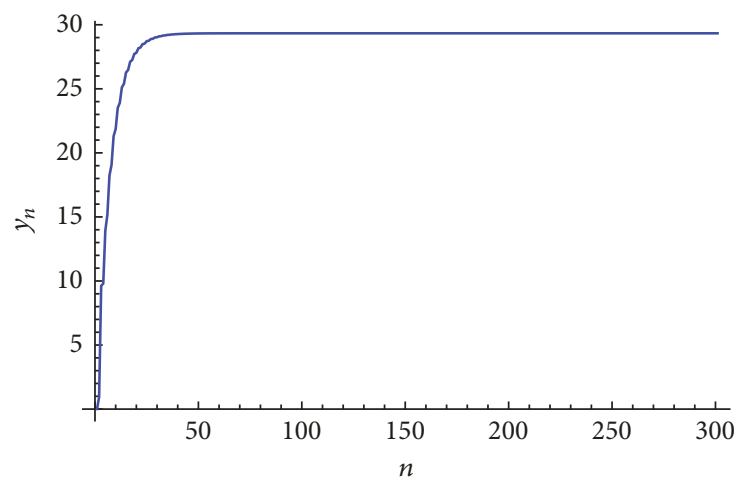

(b) Plot of $y_{n}$ for system (75)

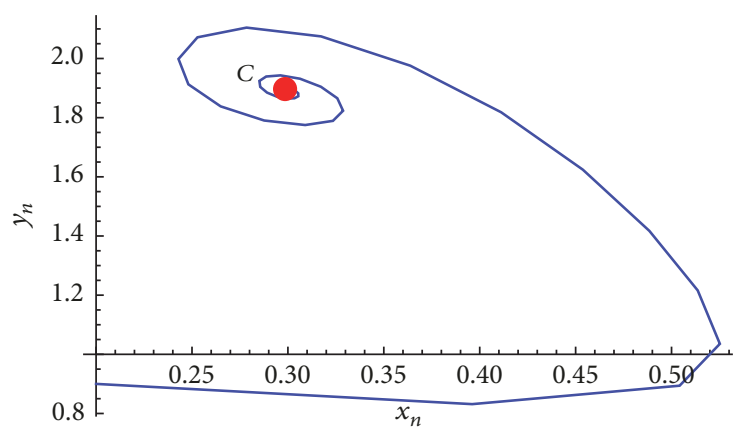

(c) Attractor of system (75)

Figure 3: Plots for system (75).

Neimark-Sacker bifurcation when parameters of the discrete model (5) are located in the following set:

$$
\begin{aligned}
N_{C} & =\left\{\left(\alpha_{1}, \alpha_{2}, \alpha_{3}, \alpha_{4}, \alpha_{5}, \alpha_{6}\right): \Delta<0, \frac{\left(\alpha_{3} \alpha_{6}-\alpha_{2} \alpha_{5}\right)^{2}-\left(\alpha_{2} \alpha_{6}\left(\alpha_{1}-1\right)-\alpha_{2}^{2}\left(\alpha_{4}-1\right)\right)\left(\alpha_{3} \alpha_{5}\left(\alpha_{4}-1\right)-\alpha_{5}^{2}\left(\alpha_{1}-1\right)\right)}{\left(\alpha_{1} \alpha_{3} \alpha_{6}-\alpha_{2} \alpha_{5}+\alpha_{2} \alpha_{3}\left(\alpha_{4}-1\right)\right)\left(\alpha_{3} \alpha_{6} \alpha_{4}-\alpha_{2} \alpha_{5}+\alpha_{6} \alpha_{5}\left(1-\alpha_{1}\right)\right)}\right. \\
& =1\} .
\end{aligned}
$$

It is proved that every positive solution of the discrete model (5) is bounded and the set $\left[0, \alpha_{1} / \alpha_{3}\right] \times\left[0, \alpha_{4} / \alpha_{6}\right]$ is an invariant rectangle. The most interesting aspect in the theory of dynamical systems is to predict the global dynamics about equilibria. In this paper, we proved that if $\alpha_{1}<1$ and $\alpha_{4}<1$, then equilibrium $O(0,0)$ of the discrete model (5) is globally asymptotically stable. Furthermore, we have investigated the global stability of the unique positive equilibrium point

$$
\begin{gathered}
C\left(\frac{\left(\alpha_{1}-1\right) \alpha_{6}-\alpha_{2}\left(\alpha_{4}-1\right)}{\alpha_{3} \alpha_{6}-\alpha_{2} \alpha_{5}},\right. \\
\left.\frac{\alpha_{3}\left(\alpha_{4}-1\right)+\alpha_{5}\left(1-\alpha_{1}\right)}{\alpha_{3} \alpha_{6}-\alpha_{2} \alpha_{5}}\right)
\end{gathered}
$$

of the discrete model (5). Some numerical examples are provided to support our theoretical results. These examples provide experimental verifications of the theoretical discussions.

\section{Conflicts of Interest}

The authors declare that they have no conflicts of interest regarding the publication of this paper.

\section{Acknowledgments}

This work was supported by the Higher Education Commission (HEC) of Pakistan.

\section{References}

[1] P. Waltman, Competition Models in Population Biology, Society for Industrial and Applied Mathematics (SIAM), 1983. 
[2] J. D. Murray, Mathematical Biology, vol. 3, Springer, New York, NY, USA, 2002.

[3] F. Brauer and C. Castillo-Chávez, Mathematical Models in Population Biology and Epidemiology, Springer, New York, NY, USA, 2001.

[4] D. Neal, Introduction to Population Biology, Cambridge University Press, Cambridge, UK, 2004.

[5] J. M. Smith, Models in Ecology, Cambridge Universty Press, 1974.

[6] H. Sedaghat, Nonlinear Difference Equations: Theory with Applications to Social Science Models, vol. 15, Kluwer Academic Publishers, Dordrecht, The Netherlands, 2003.

[7] V. L. Kocic and G. Ladas, Global Behavior of Nonlinear Difference Equations of Higher Order with Application, Kluwer Academic, Dordrecht, The Netherlands, 1993.

[8] Y. Kuang, "Global stability of Gause-type predator-prey systems," Journal of Mathematical Biology, vol. 28, no. 4, pp. 463474, 1990.

[9] S. Ahmad, "On the nonautonomous Volterra-Lotka competition equations," Proceedings of the American Mathematical Society, vol. 117, no. 1, pp. 199-204, 1993.

[10] X. Tang and X. Zou, "On positive periodic solutions of LotkaVolterra competition systems with deviating arguments," Proceedings of the American Mathematical Society, vol. 134, no. 10, pp. 2967-2974, 2006.

[11] Q. Din, "Dynamics of a discrete lotka-volterra model," Advances in Difference Equations, vol. 2013, article no. 95, 2013.

[12] M. R. S. Kulenovic and G. Ladas, Dynamics of Second Order Rational Difference Equations, Chapman \& Hall, CRC, 2002.

[13] S. Elaydi, An Introduction to Difference Equations, Springer, New York, NY, USA, 3rd edition, 2005.

[14] E. A. Grove and G. Ladas, Periodicities in Nonlinear Difference Equations, Chapman \& Hall/CRC, Boca Raton, Fla, USA, 2004.

[15] J. F. Selgrade and M. Ziehe, "Convergence to equilibrium in a genetic model with differential viability between the sexes," Journal of Mathematical Biology, vol. 25, no. 5, pp. 477-490, 1987.

[16] S. Kalabušić, M. R. S. Kulenović, and E. Pilav, "Dynamics of a two-dimensional system of rational difference equations of Leslie-Gower type," Advances in Difference Equations, vol. 2011, article no. 29, 2011.

[17] Z. Zhou and X. Zou, "Stable periodic solutions in a discrete periodic logistic equation," Applied Mathematics Letters, vol. 16, no. 2, pp. 165-171, 2003.

[18] X. Liu, "A note on the existence of periodic solutions in discrete predator-prey models," Applied Mathematical Modelling, vol. 34, no. 9, pp. 2477-2483, 2010.

[19] J. Guckenheimer and P. Holmes, Nonlinear Oscillations, Dynamical Systems, and Bifurcation of Vector Fields, Springer, New York, NY, USA, 1983.

[20] Y. A. Kuznetsov, Elements of Applied Bifurcation Theory, vol. 112 of Applied Mathematical Sciences, Springer, New York, NY, USA, 3rd edition, 2004.

[21] X. Liu and D. Xiao, "Complex dynamic behaviors of a discretetime predator-prey system," Chaos, Solitons \& Fractals, vol. 32, no. 1, pp. 80-94, 2007.

[22] A. Q. Khan, J. Ma, and D. Xiao, "Bifurcations of a twodimensional discrete time plant-herbivore system," Communications in Nonlinear Science and Numerical Simulation, vol. 39, pp. 185-198, 2016.
[23] A. Q. Khan, J. Ma, and D. Xiao, "Global dynamics and bifurcation analysis of a host-parasitoid model with strong Allee effect," Journal of Biological Dynamics, vol. 11, no. 1, pp. 121-146, 2017. 


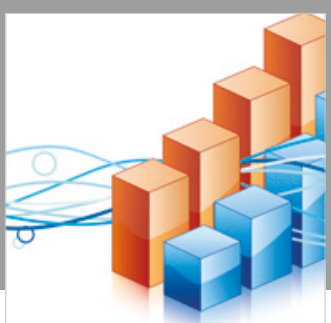

Advances in

Operations Research

\section{-n-m}
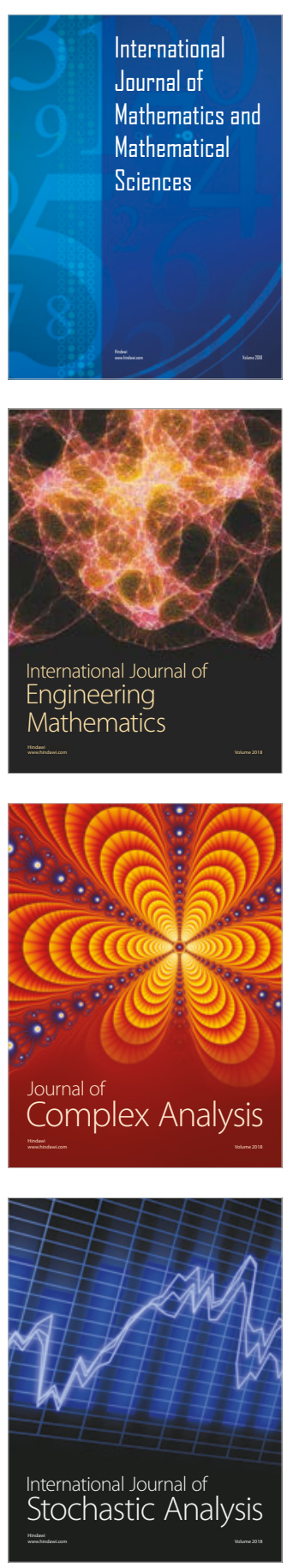
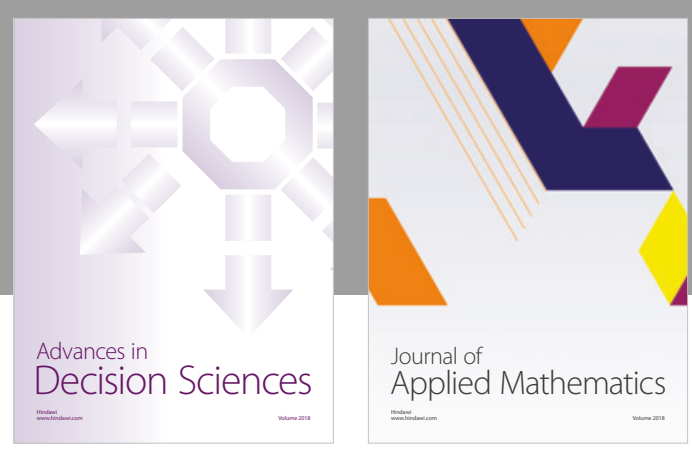

Journal of

Applied Mathematics
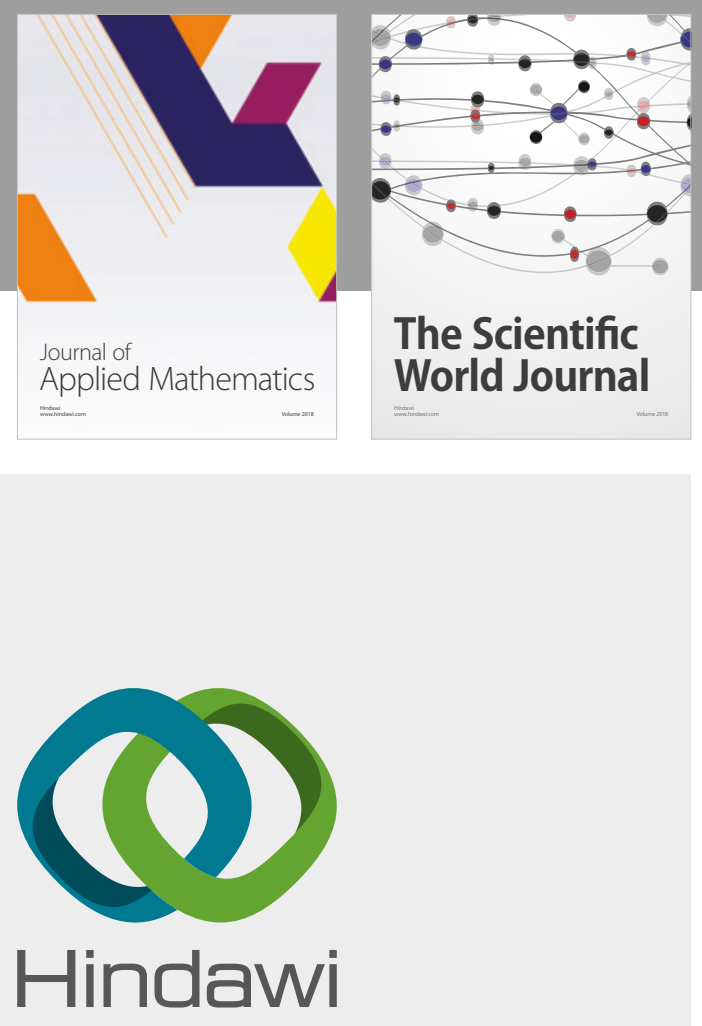

Submit your manuscripts at

www.hindawi.com

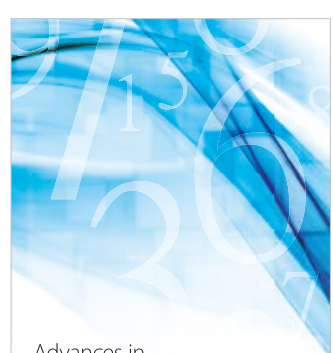

Advances in
Numerical Analysis
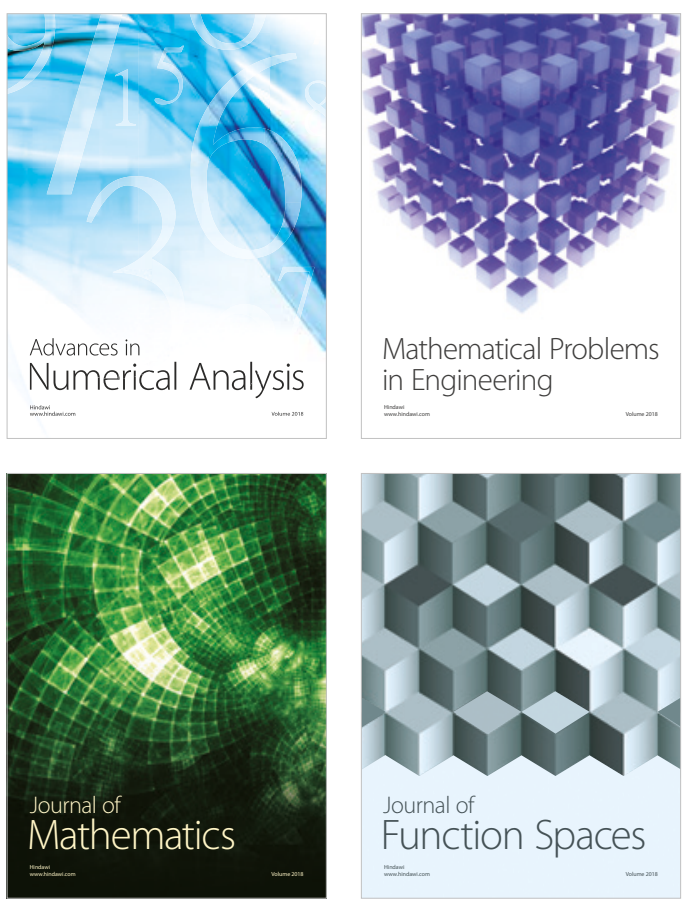

Mathematical Problems in Engineering

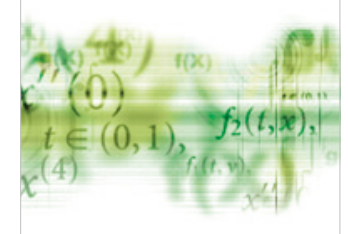

International Journal of

Differential Equations

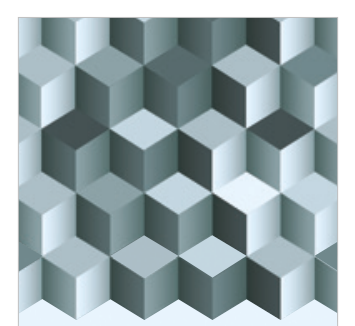

Journal of

Function Spaces
The Scientific

World Journal

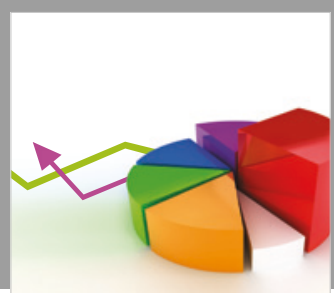

Journal of

Probability and Statistics
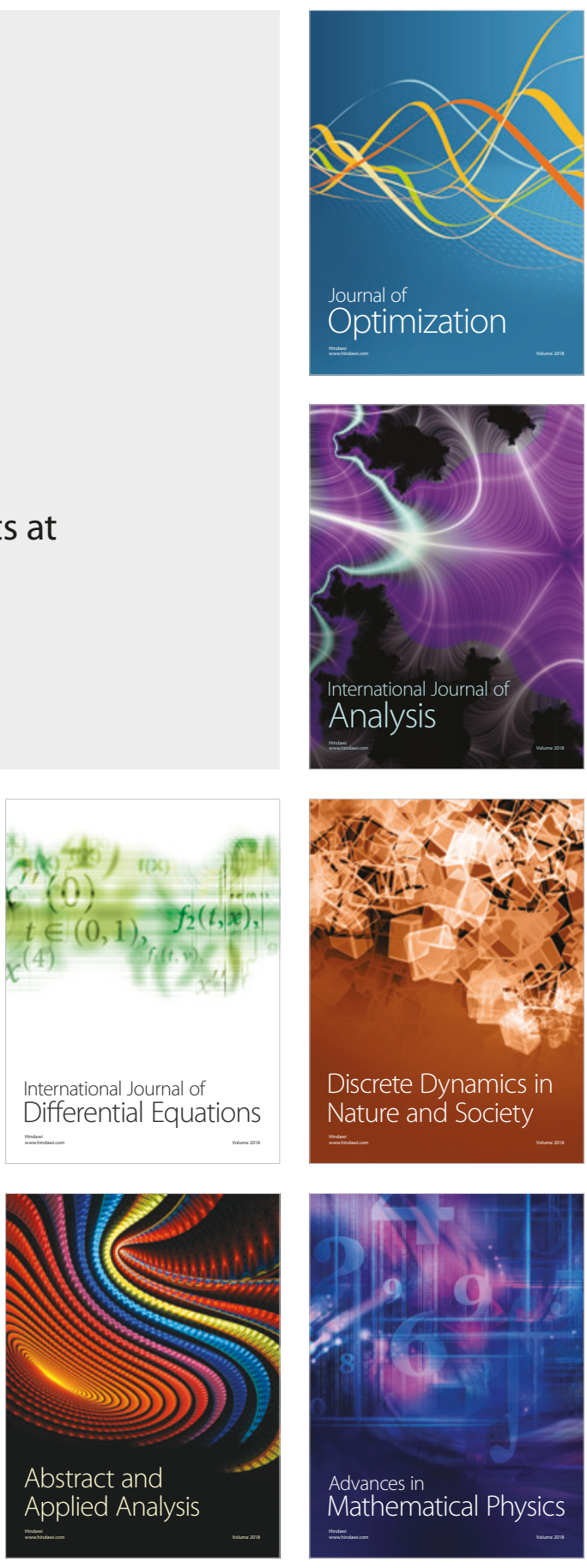